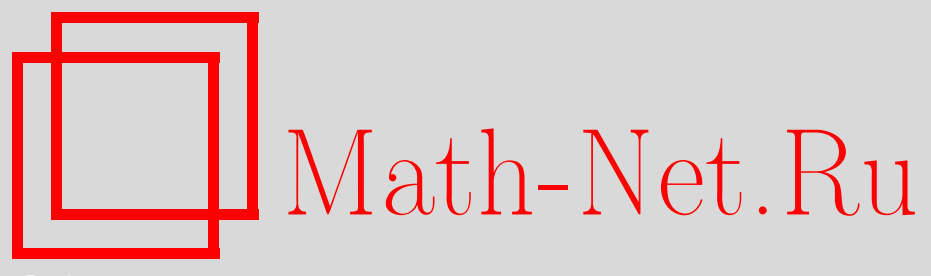

А. М. Гайнутдинов, А. М. Семихатов, И. Ю. Типунин, Б. Л. Фейгин, Соответствие Каждана-Люстига для категории представлений триплетной $W$-алгебры в логарифмических конформных теориях поля, ТМФ, 2006, том 148, номер 3, 398-427

DOI: https://doi.org/10.4213/tmf2324

Использование Общероссийского математического портала Math-Net.Ru подразумевает, что вы прочитали и согласны с пользовательским соглашением http://www.mathnet.ru/rus/agreement

Параметры загрузки:

IP: 34.229 .108 .108

26 апреля 2023 г., 10:46:40

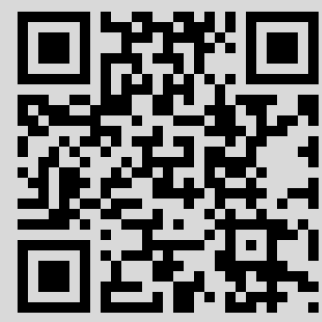




\title{
СООТВЕТСТВИЕ КАЖДАНА-ЛЮСТИГА ДЛЯ КАТЕГОРИИ ПРЕДСТАВЛЕНИЙ ТРИПЛЕТНОЙ $W$-АЛГЕБРЫ В ЛОГАРИФМИЧЕСКИХ КОНФОРМНЫХ ТЕОРИЯХ ПОЛЯ
}

\begin{abstract}
Для изучения категории представлений триплетной $W$-алгебры $\mathcal{W}(p)$, являющейся алгеброй симметрий $(1, p)$-логарифмических моделей конформной теории поля, предлагается эквивалентная категория $\mathcal{C}_{p}$ конечномерных представлений "ограниченной” квантовой группы $\overline{\mathcal{U}}_{\mathfrak{q}} s \ell(2)$ при $\mathfrak{q}=e^{i \pi / p}$. Категория $\mathcal{C}_{p}$ полностью описана посредством классификации всех неразложимых представлений, которые исчерпываются проективными модулями и тремя сериями представлений, описываемых неразложимыми представлениями колчана Кронекера. Эквивалентность категорий $\mathcal{W}(p)$ - и $\overline{\mathcal{U}}_{\mathfrak{q}} s \ell(2)$-представлений, которая предполагается для всех $p \geqslant 2$ и доказана при $p=2$, приводит к отождествлению центров квантовой группы и логарифмической конформной теории поля, а также к отождествлению универсальной $R$-матрицы и матрицы сплетения.
\end{abstract}

Ключевые слова: соответствие Каждана-Люстига, квантовые группы, логарифмические конформные теории, неразложимые представления.

\section{1. ВВЕДЕНИЕ}

1.0. Соответствие Каждана-Люстига [1] между вертекс-операторными алгебрами (конформными теориями поля) и квантовыми группами, хотя и функториальное, не является непременно эквивалентностью (а также, строго говоря, не обязательно является соответствием с категорией представлений квантовой групnы; роль последней нередко играет квазитензорная категория [2], не являющаяся категорией

*Физический факультет Московского государственного университета, Москва, Россия. E-mail: azot@mccme.ru

${ }^{\dagger}$ Физический институт им. П. Н. Лебедева РАН, Москва, Россия. E-mail: asemikha@td.lpi.ru

${ }^{\ddagger}$ Институт теоретической физики им. Л. Д. Ландау РАН, Москва, Россия. E-mail: feigin@mccme.ru 
представлений какой бы то ни было квантовой группы). "Отклонения" от эквивалентности, как правило, происходят в рациональных конформных теориях поля [3][6]. Класс моделей конформной теории поля, в которых соответствие Каждана-Люстига представляет собой эквивалентность, дается $(1, p)$-логарифмическими моделями. Это замечательное свойство можно отнести на счет того, что $(1, p)$-модели не являются логарифмическими расширениями минимальных моделей конформной теории поля, просто потому что последние не существуют. Связанная с $(1, p)$-моделью категория представлений вертекс-операторной алгебры неполупроста, и поэтому для соответствия Каждана-Люстига не требуется специального "полуупрощения" категории представлений квантовой группы. По поводу успехов в исследовании вертексоператорных алгебр с неполупростой категорией представлений см. [7], [8], а также литературу, приведенную в этих работах и в [9].

В $(1, p)$-логарифмических моделях вертекс-операторная алгебра - это триплетная $W$-алгебра $\mathcal{W}(p)$, “основанная на операторе $\phi_{3,1}$ " [10], а ее двойственная по Каждану-Люстигу квантовая группа есть $\overline{\mathcal{U}}_{\mathfrak{q}} s \ell(2)$, т.е. так называемая "ограниченная" (restricted) квантовая группа $s \ell(2)$, при $\mathfrak{q}=e^{i \pi / p}[11]$. Алгебра $\mathcal{W}(p)$ и квантовая группа $\overline{\mathcal{U}}_{\mathfrak{q}} s \ell(2)$ имеют одно и то же число $(2 p)$ неприводимых представлений и тождественные fusion-кольца (для квантовой группы - кольцо Гротендика). Результаты, полученные в [11], [12], позволяют полагать, что квазитензорные категории $\mathcal{W}(p)$ и $\overline{\mathcal{U}}_{\mathfrak{q}} s \ell(2)$-представлений в действительности эквивалентны.

В данной работе мы докажем эквивалентность в наиболее явном случае $p=2$. Доказательство обобщается на произвольные $p$, но требует более тщательного исследования, технические детали которого будут опубликованы отдельно. Общая стратегия состоит в построении бимодуля (правого $\overline{\mathcal{U}}_{\mathfrak{q}} s \ell(2)$-модуля и левого $\mathcal{W}(p)$ модуля), который разлагается на проективные модули с каждой стороны и тем самым устанавливает эквивалентность двух категорий представлений. При $p=2$ эта конструкция особенно проста, поскольку участвующие в ней вертексные операторы "почти локальны" по отношению друг к другу.

Эквивалентность, которую мы предполагаем имеющей место для всех $p$, позволяет заменить изучение категории $\mathcal{W}(p)$-представлений изучением категории конечномерных $\overline{\mathcal{U}}_{\mathfrak{q}} s \ell(2)$-представлений. Последние несложно классифицировать, используя стандартные средства [13]; как мы покажем, помимо проективных модулей, все неразложимые представления перечисляются в терминах неразложимых представлений колчана Кронекера и легко могут быть построены явно; имеются четыре серии, пронумерованные целым $n \in \mathbb{Z} \geqslant 2$, и две серии, пронумерованные посредством $\left(n \in \mathbb{Z}_{\geqslant 1}, z \in \mathbb{C P}^{1}\right)$. В силу эквивалентности это же относится и к представлениям алгебры $\mathcal{W}(p)$, прямая классификация которых представляется существенно более сложной задачей.

Эквивалентность категорий приводит к ряду отождествлений между “данными конформной теории поля" и "квантово-групповыми данными", и весьма интересным является отождествление соответствующих центров. Центр $\mathfrak{Z}_{\mathrm{ct}}$ модели конформной теории поля - эндоморфизмы тождественного функтора в категории представлений соответствующей вертекс-операторной алгебры $\mathcal{A}$ - содержит "сильно нелокальные" операторы (состояния Ишибаши). Естественный базис в центре строится 
в терминах конформных блоков на торе. В полупростом случае $\mathfrak{Z}_{\mathrm{ct}}$ совпадает с пространством характеров конформной теории поля, но в логарифмических моделях $\mathfrak{Z}_{\mathrm{ctt}}$ не исчерпывается характерами. Это явление в последнее время изучалось с помощью различных методов [7], [14]-[16]. Общий подход к нахождению недостающих "расширенных характеров", основанный на построении псевдоследов для класса вертекс-операторных алгебр с неполупростой категорией представлений, предложен в [7]. Расширенные характеры наряду с обычными являются решениями некоторых дифференциальных уравнений [16].

Коль скоро функтор Каждана-Люстига устанавливает эквивалентность между категориями представлений вертекс-операторной алгебры и квантовой группы, $\mathfrak{Z}_{\mathrm{cft}}$ канонически отождествляется с центром $\mathfrak{Z}$ квантовой группы. Более того, по крайней мере в $(1, p)$-моделях, это отождествление между $\mathfrak{Z}_{\mathrm{ctt}}$ и $\mathfrak{Z}$ продолжается на представления модулярной группы: $S L(2, \mathbb{Z})$-представление на $\mathfrak{Z}_{\mathrm{cft}}$, индуцированное преобразованиями $\tau \mapsto-1 / \tau$ и $\tau \mapsto \tau+1$, эквивалентно [11] $S L(2, \mathbb{Z})$-действию на центре $\mathfrak{Z}$ квантовой группы, определяемому в весьма общих терминах ленточных сплетенных тензорных категорий [17]. Актуальное отождествление $\mathfrak{Z}_{\text {cft }}$ и $\mathfrak{Z}$ приводит к замечательному соотношению

$$
\boldsymbol{v}=e^{2 i \pi L_{0}},
$$

где $\boldsymbol{v}$ - ленточный (ribbon) элемент (лежащий в центре квантовой группы), а $L_{0}$ - нулевая мода тензора энергии-импульса. Более того, $R$-матрицу, связанную с двойственной по Каждану-Люстигу квантовой группой $\mathfrak{G}$, можно отождествить с матрицей сплетения (braiding) в конформной теории поля, причем ее действие на вертексные операторы следует из конструкции квантовой группы $\mathfrak{G}$ в терминах экранирующих операторов (скринингов) и операторов снятия контура.

Заметим, что двойственная по Каждану-Люстигу квантовая группа $\mathfrak{G}$ сама по себе не является квазитреугольной (не обладает универсальной $R$-матрицей), по крайней мере в известных примерах логарифмических $(1, p)$-моделей [11] и $\left(p^{\prime}, p\right)$ моделей. Но универсальная $R$-матрица существует для несколько большей квантовой группы, порожденной генераторами из $\mathfrak{G}$, a также квадратным корнем $k$ из картановского генератора $K$ в $\mathfrak{G}\left(k^{2}=K\right)$. В конформной теории поля аналог произвола в определении $k=\sqrt{K}$ состоит в произволе выбора направления контуров, по которым переставляются операторы. Это приводит к двум матрицам сплетения $B^{+}$ и $B^{-}$, хорошо известным в (рациональных) конформных теориях поля [3]-[5], [15].

1.1. Определение. Пусть $\overline{\mathcal{U}}_{\mathfrak{q}} s \ell(2)$ - ограниченная квантовая группа $s \ell(2)$ при $\mathfrak{q}=e^{i \pi / p}$, порожденная элементами $E, F$ и $K$ с соотношениями

$$
E^{p}=F^{p}=0, \quad K^{2 p}=\mathbf{1}
$$

и со структурой алгебры Хопфа, задаваемой как

$$
\begin{array}{cl}
K E K^{-1}=\mathfrak{q}^{2} E, \quad K F K^{-1}=\mathfrak{q}^{-2} F, \quad[E, F]=\frac{K-K^{-1}}{\mathfrak{q}-\mathfrak{q}^{-1}}, \\
\Delta(E)=\mathbf{1} \otimes E+E \otimes K, \quad \Delta(F)=K^{-1} \otimes F+F \otimes \mathbf{1}, \quad \Delta(K)=K \otimes K,
\end{array}
$$




$$
\begin{gathered}
\epsilon(E)=\epsilon(F)=0, \quad \epsilon(K)=1, \\
S(E)=-E K^{-1}, \quad S(F)=-K F, \quad S(K)=K^{-1} .
\end{gathered}
$$

Эта квантовая группа имеет $2 p$ неэквивалентных неприводимых представлений, которые мы обозначаем как $X_{s}^{+}$и $X_{s}^{-}, 1 \leqslant s \leqslant p$. Модуль $X_{s}^{ \pm}$имеет старший вес $\pm \mathfrak{q}^{s-1}$, при этом $\operatorname{dim} X_{s}^{ \pm}=s$.

Пусть $\mathcal{C}_{p}-$ категория конечномерных $\mathbb{Z}$-градуированных $\overline{\mathcal{U}}_{\mathfrak{q}} s \ell(2)$-модулей при $\mathfrak{q}=e^{i \pi / p}$. Она стандартным образом наделяется структурой ленточной сплетенной тензорной категории (необходимый для этого ленточный элемент вычисляется по стандартному рецепту из найденной в [11] универсальной $R$-матрицы для квантовой группы, содержащей $\overline{\mathcal{U}}_{\mathfrak{q}} s \ell(2)$ в качестве подалгебры; оказывается, что результат дает ленточный элемент именно для $\overline{\mathcal{U}}_{\mathfrak{q}} s \ell(2)$, а не для этой большей квантовой группы).

1.2. Алгебра $\mathcal{W}(p)$. Пусть $\varphi(z)$ - скалярное поле с операторным произведением

$$
\varphi(z) \varphi(w)=\ln (z-w)
$$

тогда алгебра Вирасоро с центральным зарядом $c=13-6(p+1 / p)$ порождается тензором энергии-импульса (подразумевается нормальное упорядочение)

$$
T=\frac{1}{2} \partial \varphi \partial \varphi+\frac{\alpha_{0}}{2} \partial^{2} \varphi
$$

где $\alpha_{+}=\sqrt{2 p}, \alpha_{-}=-\sqrt{2 / p}$ и $\alpha_{0}=\alpha_{+}+\alpha_{-}$. Имеем скрининг-операторы

$$
S_{+}=\frac{1}{2 i \pi} \oint d u e^{\alpha_{+} \varphi(u)}, \quad S_{-}=\frac{1}{2 i \pi} \oint d u e^{\alpha_{-} \varphi(u)}
$$

и $W$-алгебру $\mathcal{W}(p)$ [10], порожденную тремя токами

$$
W^{-}(z)=\phi_{3,1}(z) \equiv e^{-\alpha_{+} \varphi}(z), \quad W^{0}(z)=\left[S_{+}, W^{-}(z)\right], \quad W^{+}(z)=\left[S_{+}, W^{0}(z)\right]
$$

(которые суть примарные поля относительно алгебры Вирасоро с конформными размерностями $2 p-1$ ).

Имеется $2 p$ неприводимых $\mathcal{W}(p)$-модулей, которые мы обозначаем как $\mathfrak{X}_{s}^{+}$("синглеты", связанные с оператором $\phi_{1, s}$ ) и $\mathfrak{X}_{s}^{-}$("дублеты", связанные с оператором $\phi_{2, s}$ ), $1 \leqslant s \leqslant p$, характеры которых имеют вид [12]

$$
\begin{aligned}
& \chi_{\mathfrak{X}_{s}^{+}}(q)=\frac{1}{\eta(q)}\left(\frac{s}{p} \theta_{p-s, p}(q)+2 \theta_{p-s, p}^{\prime}(q)\right), \quad 1 \leqslant s \leqslant p, \\
& \chi_{\mathfrak{X}_{s}^{-}}(q)=\frac{1}{\eta(q)}\left(\frac{s}{p} \theta_{s, p}(q)-2 \theta_{s, p}^{\prime}(q)\right),
\end{aligned}
$$

3 Теоретическая и математическая физика, т. 148, № 3, 2006 г. 
где

$$
\eta(q)=q^{\frac{1}{24}} \prod_{n=1}^{\infty}\left(1-q^{n}\right), \quad \theta_{s, p}(q, z)=\sum_{j \in \mathbb{Z}+s /(2 p)} q^{p j^{2}} z^{j}
$$

и мы полагаем $\theta_{s, p}(q)=\theta_{s, p}(q, 1)$ и $\theta_{s, p}^{\prime}(q)=\left.z \frac{\partial}{\partial z} \theta_{s, p}(q, z)\right|_{z=1}$. Правила слияния для $\mathcal{W}(2)$ были непосредственно вычислены в [18] (см. также [12] для $\mathcal{W}(p))$.

Подходящим выбором категории представлений для $\mathcal{W}(p)$ является категория $\mathcal{O}$.

1.3. Определение. Пусть $\mathfrak{C}_{p}$ обозначает категорию $\mathcal{O}$ представлений алгебры $\mathcal{W}(p)$, т.е. категорию модулей, в которых присоединенные собственные пространства оператора $L_{0}$ конечномерны, а спектр $L_{0}$ ограничен с одной стороны.

Это сплетенная ленточная квазитензорная категория; ее квазитензорная структура следует из операторного произведения в конформной теории поля, структура сплетения выводится из монодромии корреляционных функций, а ленточная структура задается оператором $e^{2 i \pi L_{0}}$.

1.4. Теорема. Категории $\mathrm{C}_{2} u \mathfrak{C}_{2}$ эквивалентны как ленточные сплетенные квазитензорные категории.

Как отмечено выше, доказательство этого утверждения значительно облегчается тем, что при $p=2$ имеются "почти локальные" по отношению друг к другу поля в теории симплектических фермионов. В общем случае доказательство требует проработки ряда технических деталей, однако имеется мало сомнений в верности следующего утверждения.

1.5. Гипотеза. Для всякого целого $p \geqslant 2$ категории $\mathfrak{C}_{p} u \mathfrak{C}_{p}$ эквивалентны как ленточные сплетенные квазитензорные категории.

Полагая, что эквивалентность имеет место для всех $p$, можно описывать категорию $\mathcal{W}(p)$-представлений, исследуя категорию $\overline{\mathcal{U}}_{\mathfrak{q}} s \ell(2)$-представлений. Это представляет собой достаточно простую задачу, которая в значительной степени сводится к описанию неразложимых представлений колчана Кронекера. Приведем теперь список неразложимых модулей, а затем в 1.7 сформулируем результат.

1.6. Неразложимые модули. Ключевой факт состоит в том, что те из пространств $\operatorname{Ext}_{\overline{\mathcal{U}}_{\mathfrak{q}}}$ расширений между неприводимыми представлениями, которые не являются нулевыми, двумерны (см. 2.3); в терминах базисов $\left\{x_{i}^{+}\right\}$и $\left\{x_{i}^{-}\right\}, i=$ 1,2 , выбранных в соответствующих пространствах $\mathbb{C}^{2}=\operatorname{Ext} \frac{1}{\bar{u}_{\mathfrak{q}}}\left(X_{s}^{+}, X_{p-s}^{-}\right)$и $\mathbb{C}^{2}=$ $\operatorname{Ext} \frac{1}{\mathcal{U}_{q}}\left(X_{p-s}^{-}, X_{s}^{+}\right)$, можно построить четыре семейства неразложимых модулей как указано ниже. В приводимых диаграммах $X_{1} \stackrel{x}{\longrightarrow} X_{2}$ обозначает расширение посредством $x \in \operatorname{Ext} \frac{1}{\overline{\mathcal{U}}_{\mathfrak{q}}}\left(X_{1}, X_{2}\right)$, причем $X_{1}$ является неприводимым подфактором, а $x_{2}$ - неприводимым подмодулем. 
Модули $\mathcal{W}_{\boldsymbol{s}}^{ \pm}(\boldsymbol{n})$. При $1 \leqslant s \leqslant p-1, a= \pm$ и целом $n \geqslant 2$ модуль $\mathcal{W}_{s}^{a}(n)$ определяется как

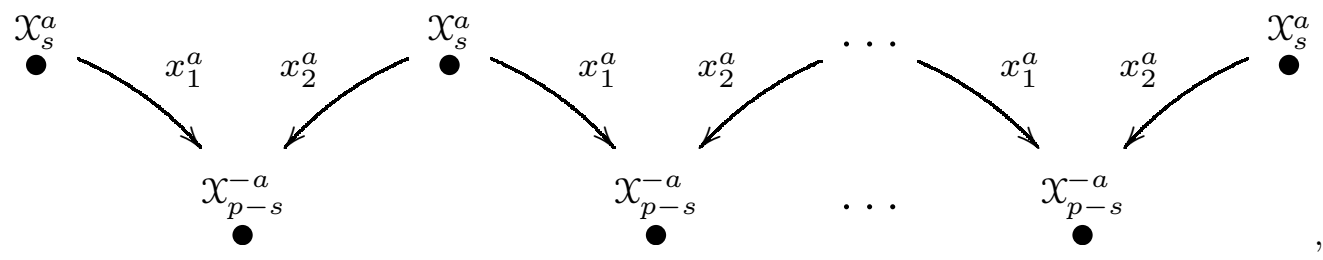

где $n$ - число модулей $X_{s}^{a}$. Базис и $\overline{\mathcal{U}}_{\mathfrak{q}} s \ell(2)$-действие на $\mathcal{W}_{s}^{a}(n)$ явно описаны в А.1.1 на примере $\mathcal{W}_{s}^{a}(2)$.

Модули $\mathcal{M}_{\boldsymbol{s}}^{ \pm}(\boldsymbol{n})$. При $1 \leqslant s \leqslant p-1, a= \pm$ и целом $n \geqslant 2$ модуль $\mathcal{M}_{s}^{a}(n)$ определяется как

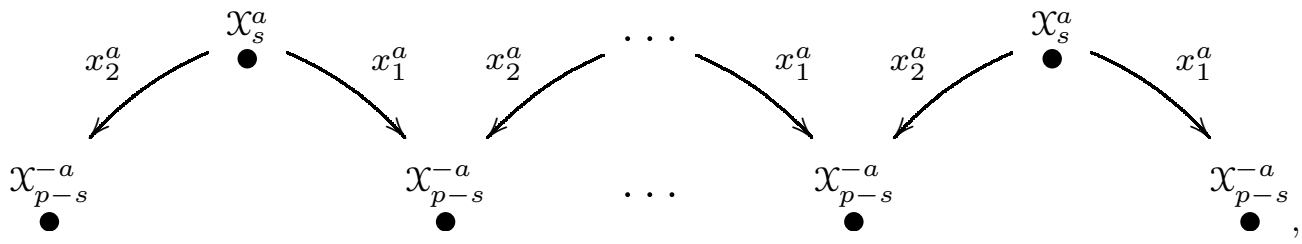

где $n$ - число модулей $\mathcal{X}_{p-s}^{-a}$. Базис и $\overline{\mathcal{U}}_{\mathfrak{q}} s \ell(2)$-действие на $\mathcal{M}_{s}^{a}(n)$ явно описаны в А.1.2 на примере $\mathcal{M}_{s}^{a}(2)$.

Модули $\mathcal{O}_{s}^{ \pm}(\boldsymbol{n}, \boldsymbol{z})$. При $1 \leqslant s \leqslant p-1, a= \pm$, целом $n \geqslant 1$ и $z \in \mathbb{C P}^{1}$ модуль $\mathcal{O}_{s}^{a}(n, z)$ определяется как

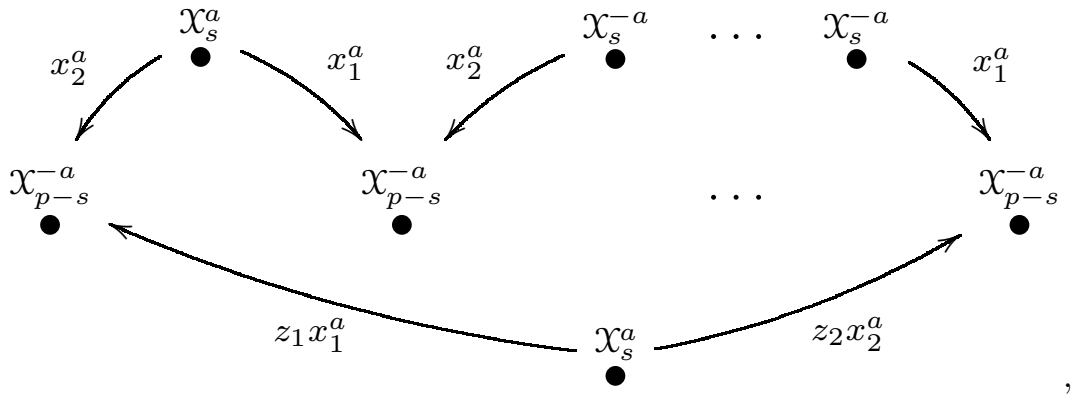

где $n$ - полное число модулей $\chi_{s}^{a}$, а $z=z_{1}: z_{2} \in \mathbb{C P}^{1}$. Базис и $\overline{\mathcal{U}}_{\mathfrak{q}} s \ell(2)$-действие на $\mathcal{O}_{s}^{a}(n, z)$ явно описаны в А.1.3 на примере $\mathcal{O}_{s}^{a}(1, z)$. 
Модули $\mathcal{P}_{s}^{ \pm}$. При $1 \leqslant s \leqslant p-1$ и $a= \pm$ проективный модуль $\mathcal{P}_{s}^{a}$ определен в А.2; его структура подфакторов (см. 2.4 ниже) описывается диаграммой

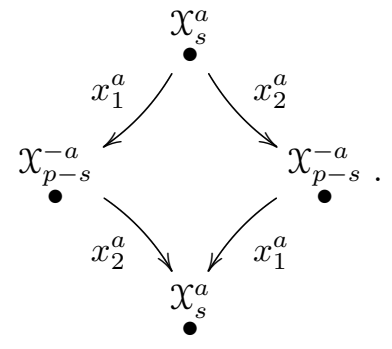

Следующая теорема показывает, что приведенный список исчерпывает конечномерные неразложимые $\overline{\mathcal{U}}_{\mathfrak{q}} s \ell(2)$-модули.

\section{7. Классификационная теорема для $\mathcal{C}_{p}$.}

1) Категория $\mathcal{C}_{p}$ имеет разложение

$$
\mathrm{e}_{p}=\bigoplus_{s=0}^{p} \mathrm{e}_{p}(s)
$$

где каждая $\mathcal{C}_{p}(s)$ является полной подкатегорией.

2) Каждая из подкатегорий $\mathcal{C}_{p}(0)$ u $\mathcal{C}_{p}(p)$ полупроста и содержит ровно один неприводимый модуль, $X_{p}^{+}$и $X_{p}^{-}$, соответственно.

3) Каждая подкатегория $\mathrm{C}_{p}(s), 1 \leqslant s \leqslant p-1$, содержит ровно два неприводимых модуля $X_{s}^{+}$и $X_{p-s}^{-}$и следующий набор неразложимых модулей:

- проективные модули $\mathcal{P}_{s}^{+}$u $\mathcal{P}_{p-s}^{-}$;

- три серии неразложимых модулей:

- модули $\mathcal{W}_{s}^{+}(n)$ и $\mathcal{W}_{p-s}^{-}(n)$ для всех иельх $n \geqslant 2$,

- модули $\mathcal{M}_{s}^{+}(n)$ и $\mathcal{M}_{p-s}^{-}(n)$ для всех целых $n \geqslant 2$,

- модули $\mathcal{O}_{s}^{+}(n, z)$ и $\mathcal{O}_{p-s}^{-}(n, z)$ для всех $z \in \mathbb{C P}^{1}$ и иелых $n \geqslant 1$.

Этим исчерпьвается список неразложимых модулей в $\mathcal{C}_{p}(s)$.

Далее, для каждой подкатегории $\mathcal{C}_{p}(s)$ при $1 \leqslant s \leqslant p-1$ векторное пространство

$$
\mathrm{Ext}_{s}^{\bullet}=\bigoplus_{n \geqslant 0} \operatorname{Ext}_{\overline{\mathrm{u}}_{\mathfrak{q}}}^{n}\left(X_{s}^{+} \oplus \mathcal{X}_{p-s}^{-}, X_{s}^{+} \oplus \mathcal{X}_{p-s}^{-}\right)
$$

является ассоциативной алгеброй относительно произведения Йонеды. В следующей теореме мы описываем алгебраическую структуру $\mathrm{Ext}_{s}^{\bullet}$.

1.8. Теорема. Алгебра $\mathrm{Ext}_{s}^{\bullet}$ порождена элементами $x_{i}^{ \pm}, i, j=1,2$, с определяющими соотношениями

$$
x_{i}^{+} x_{j}^{+}=x_{i}^{-} x_{j}^{-}=x_{1}^{+} x_{2}^{-}+x_{2}^{+} x_{1}^{-}=x_{1}^{-} x_{2}^{+}+x_{2}^{-} x_{1}^{+}=0 .
$$

Заметим, что производная категория категории представлений алгебры $\mathrm{Ext}_{s}^{\bullet}$ эквивалентна производной категории для $\mathcal{C}_{p}(s)$ (а потому и производной категории полной подкатегории $\mathcal{W}(p)$-представлений). 
Обозначения. Положим

$$
\mathfrak{q}=e^{i \pi / p}
$$

где $p=2$ в разделе 4 и $p \in \mathbb{Z} \geqslant 2$ в остальной статье. Ниже $\mathbb{Z}_{+}$обозначает множество $\mathbb{N} \cup\{0\}$.

Данная работа построена следующим образом. Доказательство классификационного результата 1.7 дано в разделе 2. В разделе 3 доказана теорема 1.8. Эквивалентность категорий представлений $W$-алгебры и квантовой группы доказана в разделе 4 для $p=2$. Явные конструкции некоторых модулей приведены в приложении А. Необходимые факты о колчанах сообщаются в приложении Б.

\section{2. ДОКАЗАТЕЛЬСТВО КЛАССИФИКАЦИОННОЙ ТЕОРЕМЫ}

Докажем 1.7 путем непосредственного исследования структуры категории $\mathrm{C}_{p}$. Зафиксируем целое число $p \geqslant 2$. Схема доказательства такова. В 2.2 , используя оператор Казимира, мы разложим категорию $\mathcal{C}_{p}$ в прямую сумму полных подкатегорий $\mathcal{C}_{p}(s)$. В 2.3 мы вычислим $\operatorname{Ext} \frac{1}{\overline{\mathcal{q}}_{\mathfrak{q}}}$ между неприводимыми представлениями в каждой $\mathcal{C}_{p}(s)$. В 2.4 строятся проективные модули в каждой полной подкатегории $\mathcal{C}_{p}(s)$. Далее заметим, что все проективные модули в $\mathcal{C}_{p}$ являются также инъективными. Этой информации достаточно для того, чтобы убедиться, что неразложимые модули с полупростой длиной 3 являются проективными модулями и что не существуют модули с полупростой длиной 4 или более. Поэтому для завершения доказательства 1.7 остается классифицировать неразложимые модули с полупростой длиной 2 . Это сделано в 2.5 с использованием соответствия между модулями с полупростой длиной 2 и неразложимыми представлениями колчана Кронекера.

2.1. Некоторые соглашения и определения. В отношении обозначений и общих фактов о $\overline{\mathcal{U}}_{\mathfrak{q}} \equiv \overline{\mathcal{U}}_{\mathfrak{q}} s \ell(2)$, которые потребуются ниже, мы следуем главным образом работе [11].

2.1.1. Ограниченная квантовая группа $\overline{\mathcal{U}}_{\mathfrak{q}} s \ell(2)$. Алгебра Хопфа $\overline{\mathcal{U}}_{\mathfrak{q}} s \ell(2)$ порождена элементами $E, F$ и $K$ с соотношениями, приведенными в 1.1. Во всех $\bar{U}_{\mathfrak{q}} s \ell(2)$-модулях выберем базисы так, чтобы генератор $K$ действовал диагонально (действие $K$ на всяком модуле из $\mathcal{C}_{p}$ диагонализуемо, потому что $K^{2 p}=\mathbf{1}$ ).

Пусть $\mathfrak{Z}$ обозначает центр $\overline{\mathcal{U}}_{\mathfrak{q}} s \ell(2)$ (этот центр $(3 p-1)$-мерен [11]).

2.1.2. Неприводимые модули. Неприводимые $\overline{\mathcal{U}}_{\mathfrak{q}} s \ell(2)$-модули пронумерованы своими старшими весами $\mathfrak{q}^{s-1}$, где $s \in \mathbb{Z} / 2 p \mathbb{Z}$. Мы будем также параметризовать те же старшие веса как $a \mathfrak{q}^{s-1}$, где $a= \pm$ и $1 \leqslant s \leqslant p$. Тогда при $1 \leqslant s \leqslant p$ неприводимый модуль со старшим весом $\pm \mathfrak{q}^{s-1}$ обозначается как $\chi_{s}^{ \pm}$. Модуль $\chi_{s}^{ \pm}$размерности $s$ линейно порожден элементами $\mathrm{a}_{n}^{ \pm}, 0 \leqslant n \leqslant s-1$, где $\mathrm{a}_{0}^{ \pm}-$вектор старшего веса, а действие алгебры задается как

$$
\begin{aligned}
K \mathrm{a}_{n}^{ \pm} & = \pm \mathfrak{q}^{s-1-2 n} \mathrm{a}_{n}^{ \pm}, \\
E \mathrm{a}_{n}^{ \pm} & = \pm[n][s-n] \mathrm{a}_{n-1}^{ \pm}, \\
F \mathrm{a}_{n}^{ \pm} & =\mathrm{a}_{n+1}^{ \pm},
\end{aligned}
$$


при этом мы полагаем $\mathrm{a}_{-1}^{ \pm}=\mathrm{a}_{s}^{ \pm}=0$ и используем стандартное обозначение

$$
[n]=\frac{\mathfrak{q}^{n}-\mathfrak{q}^{-n}}{\mathfrak{q}-\mathfrak{q}^{-1}} .
$$

Модуль $X_{1}^{+}$является тривиальным.

2.1.3. Расширения. Пусть $A$ и $C$-левые $\overline{\mathcal{U}}_{\mathfrak{q}} s \ell(2)$-модули. Будем говорить, что короткая точная последовательность $\overline{\mathcal{U}}_{\mathfrak{q}} s \ell(2)$-модулей $0 \rightarrow A \rightarrow B \rightarrow C \rightarrow 0$, или эквивалентно,

$$
A \longmapsto B \rightarrow C,
$$

является расширением $C$ с помощью $A$. Пусть $\operatorname{Ext}_{\overline{\mathcal{u}}_{\mathfrak{q}}}(C, A)$ обозначает множество классов эквивалентности (см., например, [19]) расширений $C$ с помощью $A$.

2.1.4. Полупростая длина модуля. Пусть $\mathcal{N}-\overline{\mathcal{U}}_{\mathfrak{q}} s \ell(2)$-модуль. Определим полупростую фильтрацию модуля $\mathcal{N}$ как башню подмодулей

$$
\mathcal{N}=\mathcal{N}_{0} \supset \mathcal{N}_{1} \supset \ldots \supset \mathcal{N}_{l}=0,
$$

где каждый фактор $\mathcal{N}_{i} / \mathcal{N}_{i+1}$ полупрост. Число $l$ назовем длиной фильтрации. На множестве полупростых фильтраций $\mathcal{N}$ существует фильтрация с минимальной длиной $\ell$. Назовем $\ell$ полупростой длиной модуля $\mathcal{N}$.

Очевидно, полупростая длина неразложимого модуля не меньше 2. Всякий полупростой модуль имеет полупростую длину 1.

2.2. Разложение категории $\mathcal{C}_{p}$. Напомним, что элемент Казимира

$$
\boldsymbol{C}=E F+\frac{\mathfrak{q}^{-1} K+\mathfrak{q} K^{-1}}{\left(\mathfrak{q}-\mathfrak{q}^{-1}\right)^{2}}=F E+\frac{\mathfrak{q} K+\mathfrak{q}^{-1} K^{-1}}{\left(\mathfrak{q}-\mathfrak{q}^{-1}\right)^{2}} \in \mathfrak{Z}
$$

удовлетворяет минимальному полиномиальному соотношению (см., например, [11])

$$
\Psi_{2 p}(\boldsymbol{C})=0
$$

где

$$
\Psi_{2 p}(x)=\left(x-\beta_{0}\right)\left(x-\beta_{p}\right) \prod_{j=1}^{p-1}\left(x-\beta_{j}\right)^{2}, \quad \beta_{j}=\frac{\mathfrak{q}^{j}+\mathfrak{q}^{-j}}{\left(\mathfrak{q}-\mathfrak{q}^{-1}\right)^{2}},
$$

а потому порождает подалгебру в $\mathfrak{Z}$ размерности $2 p$. Это соотношение дает такое разложение категории $\mathcal{C}_{p}$ в прямую сумму полных подкатегорий $\mathcal{C}_{p}(j)$, что $\left(\boldsymbol{C}-\beta_{j}\right)$ действует нильпотентно на объектах из $\mathcal{C}_{p}(j)$. Поскольку $\beta_{j} \neq \beta_{j^{\prime}}$ при $0 \leqslant j \neq$ $j^{\prime} \leqslant p$, имеется $p+1$ полных подкатегорий $\mathcal{C}_{p}(s), 0 \leqslant s \leqslant p$. Этим доказана часть 1 теоремы 1.7 .

Каждая подкатегория $\mathcal{C}_{p}(s)$ при $1 \leqslant s \leqslant p-1$ содержит ровно два неприводимых модуля $X_{s}^{+}$и $X_{p-s}^{-}$, потому что элемент Казимира действует умножением на $\beta_{s}$ в точности на этих двух неприводимых модулях. Неприводимые модули $X_{p}^{+}$и $X_{p}^{-}$, отвечающие соответствующим собственным значениям $\beta_{p}$ и $\beta_{0}$, принадлежат к соответствующим категориям $\mathcal{C}_{p}(p)$ и $\mathcal{C}_{p}(0)$. 
Для описания классов изоморфизмов неразложимых представлений опишем сначала расширения между неприводимыми представлениями. Непосредственный анализ показывает, что $\operatorname{Ext} \frac{1}{\mathcal{u}_{\mathfrak{q}}}\left(\mathcal{X}_{s}^{a}, \mathcal{X}_{p-s}^{-a}\right) \cong \mathbb{C}^{2}$, а все остальные $\operatorname{Ext} \frac{1}{\overline{\mathcal{u}}_{\mathfrak{q}}}$ между неприводимыми представлениями в $\mathcal{C}_{p}(s)$ обращаются в нуль. Таким образом имеем следующую лемму.

2.3. Лемма. Для $1 \leqslant s \leqslant p$ u $a, a^{\prime}= \pm$ имеются изоморфизмы векторных пространсте

$$
\operatorname{Ext} \frac{1}{\bar{u}_{\mathfrak{q}}}\left(X_{s}^{a}, X_{s^{\prime}}^{a^{\prime}}\right) \cong \begin{cases}\mathbb{C}^{2} & \text { при } a^{\prime}=-a, s^{\prime}=p-s \\ 0 & \text { в остальных случалх }\end{cases}
$$

Кроме того, видно, что часть 2 в теореме 1.7 о неприводимых модулях из $\mathcal{C}_{p}(s)$ выполнена.

2.3.1. Замечание. Пространство $\mathbb{C}^{2}$ в этой лемме является фундаментальным представлением алгебры Ли $s \ell(2)$, посредством которой $\overline{\mathcal{U}}_{\mathfrak{q}} s \ell(2)$ расширяется до квантовой группы Люстига "с разделенными степенями". Это есть в действительности еще одно проявление эквивалентности категорий $\overline{\mathcal{U}}_{\mathfrak{q}} s \ell(2)-$ и $\mathcal{W}(p)$-представлений: появление алгебры Ли $s \ell(2)$ хорошо известно в $(1, p)$-моделях конформной теории поля, где она является внешним автоморфизмом триплетной $W$-алгебры $\mathcal{W}(p)$, а потому действует на некоторых $\mathcal{W}(p)$-представлениях, и в частности, на расширениях двух неприводимых представлений.

2.3.2. Модули Верма и базисы в $\mathbb{C}^{2}$. Для каждого $1 \leqslant s \leqslant p$ и $a= \pm$ пусть $\mathcal{V}_{s}^{a}$ обозначает модуль Верма со старшим весом $a \mathfrak{q}^{s-1}$, и пусть $\overline{\mathcal{V}}_{s}^{a}-$ контрагредиентный модуль Верма со старшим весом $a \mathfrak{q}^{-s-1}$ (он является контрагредиентным к модулю Верма $\left.\mathcal{V}_{p-s}^{-a}\right)^{1)}$. Заметим, что $\mathcal{V}_{p}^{ \pm}=\overline{\mathcal{V}}_{p}^{ \pm}=X_{p}^{ \pm}$.

Для каждого $1 \leqslant s \leqslant p-1$ выберем базис $\left\{x_{i}^{+}\right\}$и $\left\{x_{i}^{-}\right\}, i=1,2$, в соответствующем пространстве $\mathbb{C}^{2}=\operatorname{Ext} \frac{1}{\mathcal{U}_{q}}\left(X_{s}^{+}, X_{p-s}^{-}\right)$и $\mathbb{C}^{2}=\operatorname{Ext} \frac{1}{\overline{\mathcal{U}}_{q}}\left(X_{p-s}^{-}, X_{s}^{+}\right)$так, чтобы $x_{1}^{+}$соответствовало модулю Верма $\mathcal{V}_{s}^{+}, x_{2}^{+}$соответствовало контрагредиентному модулю Верма $\overline{\mathcal{V}}_{s}^{+}, x_{1}^{-}$соответствовало $\mathcal{V}_{p-s}^{-}$, а $x_{2}^{-}$соответствовало $\overline{\mathcal{V}}_{p-s}^{-}$.

2.3.3. Замечание. В терминах действия $\overline{\mathcal{U}}_{\mathfrak{q}} s \ell(2)$-генераторов $E$ и $F$ расширение $X_{s}^{+} \stackrel{x_{1}^{+}}{\longrightarrow} X_{p-s}^{-}$, дающее модуль Верма, устроено так, что $F$ отправляет вектор младшего веса из подфактора $X_{s}^{+}$в вектор старшего веса в подмодуле $X_{p-s}^{-}$; аналогично расширение, определяемое элементом $x_{2}^{+}$и дающее контрагредиентный модуль Верма, таково, что $E$ отправляет вектор старшего веса из $X_{s}^{+}$в вектор младшего веса в $x_{p-s}^{-}$. Расширения, определяемые элементами $x_{i}^{-}, i=1,2$, описываются аналогично.

2.4. Проективные модули. Опишем проективные модули в каждой подкатегории $\mathcal{C}_{p}(s)$ при $0 \leqslant s \leqslant p$. Для построения этих модулей используем тот факт, что всякий неразложимый проективный модуль можно получить как проективную накрывающую неприводимого модуля.

\footnotetext{
1) Модуль Верма $\mathcal{V}_{s}^{a}$ и контрагредиентный модуль Верма $\bar{V}_{s}^{a}$ являются частными случаями соответствующих $\mathcal{O}_{s}^{a}(1, z)$-модулей при $z=z_{1}: z_{2}=1: 0$ и $z=0: 1$.
} 
2.4.1. Предложение. (1) Проективными модулями в подкатегориях $\mathcal{C}_{p}(0)$ и $\mathrm{C}_{p}(p)$ являются соответствующие неприводимье модули $X_{p}^{+}$u $X_{p}^{-}$.

(2) Для каждого $s, 1 \leqslant s \leqslant p-1$, проективными модулями в подкатегории $\mathrm{C}_{p}(s)$ являются $\mathcal{P}_{s}^{+}$u $\mathcal{P}_{p-s}^{-}($см. (1.2)).

ДокАзАтЕЛЬСтво. Часть (1) очевидна, поскольку подкатегории $\mathcal{C}_{p}(0)$ и $\mathcal{C}_{p}(p)$ полупросты. Чтобы доказать часть (2), построим проективную накрывающую каждого неприводимого модуля. Сначала построим нетривиальное расширение модуля $x_{s}^{+}$максимальным числом неприводимых модулей $X_{p-s}^{-}$, т.е. расширение посредством $X_{p-s}^{-} \otimes \operatorname{Ext} \frac{1}{\overline{\mathcal{u}}_{\mathfrak{q}}}\left(X_{s}^{+}, X_{p-s}^{-}\right)$, что дает

$$
X_{p-s}^{-} \oplus X_{p-s}^{-} \longmapsto \mathcal{M}_{s}^{+}(2) \rightarrow X_{s}^{+} .
$$

Неразложимый модуль $\mathcal{M}_{s}^{+}(2)$ явно описан в А.1.2.

Далее, чтобы найти проективную накрывающую модуля $X_{s}^{+}$, расширим каждое слагаемое в подмодуле $X_{p-s}^{-} \oplus X_{p-s}^{-} \subset \mathcal{M}_{s}^{+}(2)$ посредством $X_{s}^{+} \otimes \operatorname{Ext} \frac{1}{\bar{u}_{q}}\left(X_{p-s}^{-}, X_{s}^{+}\right)$. Условия совместности с соотношениями алгебры $\overline{\mathcal{U}}_{\mathfrak{q}} s \ell(2)$ (в частности, с $F^{p}=0$ и $\left.E^{p}=0\right)$ приводят к расширению, отвечающему модулю $\mathcal{P}_{s}^{+}$, который есть проективная накрывающая модуля $X_{s}^{+}$. Этот модуль явно описан в А.2.

Подобная процедура дает проективную накрывающую $\mathcal{P}_{p-s}^{-}$модуля $\mathcal{X}_{p-s}^{-}$. Модули $\mathcal{M}_{p-s}^{-}(2)$ и $\mathcal{P}_{p-s}^{-}$описаны в А.1.2 и А.2, соответственно.

Таким образом, в каждой подкатегории $\mathcal{C}_{p}(s)$ при $1 \leqslant s \leqslant p-1$ имеются два неэквивалентных проективных модуля $\mathcal{P}_{s}^{+}$и $\mathcal{P}_{p-s}^{-}$, которые накрывают соответствующие неприводимые модули. Чтобы показать, что эти проективные накрывающие исчерпывают все неразложимые проективные модули в категории $\mathcal{C}_{p}$, вспомним, что левое регулярное представление $\mathcal{R}$ алгебры $\overline{\mathcal{U}}_{\mathfrak{q}} s \ell(2)$ является прямой суммой по всем проективным модулям с кратностью каждого модуля, равной размерности его простого фактора. Размерности простых факторов в $\mathcal{P}_{s}^{ \pm}$равны $s$ (см. А.2) и $\operatorname{dim} \mathcal{X}_{p}^{ \pm}=p$, поэтому

$$
\operatorname{Reg} \supset \bigoplus_{s=1}^{p-1} s \mathcal{P}_{s}^{+} \oplus \bigoplus_{s=1}^{p-1} s \mathcal{P}_{s}^{-} \oplus p X_{p}^{+} \oplus p X_{p}^{-}
$$

С другой стороны, подсчет размерности правой части дает $\sum_{s=1}^{p-1} s \cdot 2 p+\sum_{s=1}^{p-1} s \cdot 2 p+$ $p \cdot p+p \cdot p=2 p^{3}=\operatorname{dim} \overline{\mathcal{U}}_{\mathfrak{q}} s \ell(2)$, а потому указанное включение является равенством, и имеет место утверждение (2) предложения.

2.4.2. Замечание. Из 2.4.1 следует также, что структура подфакторов в проективных модулях именно такая, как показано в (1.2).

Далее, “двойственная" процедура построения инъективных модулей в каждой подкатегории $\mathcal{C}_{p}(s)$ показывает, что они в действительности совпадают с проективными модулями (инъективные модули являются контрагредиентными к проективным, но модуль, получаемый обращением всех стрелок в (1.2), очевидно, изоморфен исходному). Отсюда вытекает следующее предложение.

2.4.3. Предложение. Проективные модули в $\mathcal{C}_{p}$ являются инбективными. 
2.4.4. Предложение. (1) $B \mathcal{C}_{p}$ не существуют модули с полупростой длиной, большей 3 .

(2) Единственными неразложимыми модулями с полупростой длиной 3 являются проективные модули $\mathcal{P}_{s}^{ \pm}$, где $1 \leqslant s \leqslant p-1$.

ДокАЗАТЕЛЬСтво. При $1 \leqslant s \leqslant p-1$ пусть $\mathcal{N}$ - такой модуль, что $\mathcal{N}_{0} / \mathcal{N}_{1}$ из $(2.1)$ содержит простое слагаемое $X_{s}^{ \pm}$. Это означает, что имеется ненулевое отображение $\mathcal{P}_{s}^{ \pm} \rightarrow \mathcal{N}$, накрывающее $\mathcal{X}_{s}^{ \pm}$. Для отображения $\mathcal{P}_{s}^{ \pm} \rightarrow \mathcal{N}$ имеются две возможности: оно является вложением или оно имеет ненулевое ядро. В первом случае вспомним, что проективный модуль в то же время является инъективным, а потому существует прямое слагаемое в любом модуле, куда он вкладывается. Во втором случае ядро с необходимостью содержит подмодуль $\chi_{s}^{ \pm}$модуля $\mathcal{P}_{s}^{ \pm}$. Но это означает, что подфактор $\mathcal{X}_{s}^{ \pm}$модуля $\mathcal{N}$ в действительности принадлежит прямому слагаемому в $\mathcal{N}$ с полупростой длиной меньше или равной 2.

2.5. Модули с полупростой длиной 2. Для завершения доказательства части (3) в 1.7 остается классифицировать конечномерные модули с полупростой длиной $\ell=2$.

2.5.1. Категория $\mathfrak{C}_{p}^{(2)}(s)$. Для $1 \leqslant s \leqslant p-1$ пусть $\mathcal{C}_{p}^{(2)}(s)$ - полная подкатегория в $\mathcal{C}_{p}(s)$, состоящая из $\overline{\mathcal{U}}_{\mathfrak{q}} s \ell(2)$-модулей с полупростой длиной $\ell \leqslant 2$. Очевидно, каждый модуль из категорий $\mathcal{C}_{p}^{(2)}(s)$ можно получить либо как

- расширение прямой суммы неприводимых модулей $\mathcal{X}_{s}^{+}$прямой суммой неприводимых модулей $X_{p-s}^{-}$с помощью прямой суммы элементов $x^{+} \in \operatorname{Ext} \frac{1}{\mathcal{U}_{q}}\left(X_{s}^{+}, X_{p-s}^{-}\right)$, либо как

- расширение прямой суммы неприводимых модулей $\chi_{p-s}^{-}$прямой суммой неприводимых модулей $X_{s}^{+}$с помощью прямой суммы элементов $x^{-} \in \operatorname{Ext} \frac{1}{\mathcal{U}_{q}}\left(X_{p-s}^{-}, X_{s}^{+}\right)$.

Для $\mathrm{X}^{+} \in \operatorname{Ext} \frac{1}{\mathcal{u}_{q}}\left(\bigoplus_{j=1}^{m} \mathcal{X}_{s}^{+}, \bigoplus_{i=1}^{n} X_{p-s}^{-}\right)$пусть $\mathcal{J}_{\mathbf{X}^{+}}^{+}(m, n) \in \mathrm{Ob}\left(\mathcal{C}_{p}^{(2)}(s)\right)$ обозначает модуль, определяемый расширением

$$
\bigoplus_{j=1}^{m} x_{s}^{+} \stackrel{\mathrm{x}^{+}}{\longrightarrow} \bigoplus_{i=1}^{n} x_{p-s}^{-} .
$$

Для $\mathrm{X}^{-} \in \operatorname{Ext}_{\overline{\mathcal{U}}_{\mathfrak{q}}}\left(\bigoplus_{j=1}^{m} \mathcal{X}_{p-s}^{-}, \bigoplus_{i=1}^{n} \mathcal{X}_{s}^{+}\right)$определим модули $\mathcal{J}_{\mathbf{X}^{-}}^{-}(m, n)$ аналогично. Положим также $\mathcal{J}_{0}^{ \pm}(m, 0)=\bigoplus_{j=1}^{m} X_{s}^{+}$и $\mathcal{J}_{0}^{ \pm}(0, n)=\bigoplus_{j=1}^{n} X_{p-s}^{-}{ }^{2}$.

Определим две полные подкатегории $\mathcal{C}_{p}^{(2),+}(s)$ и $\mathcal{C}_{p}^{(2),-}(s)$ в $\mathcal{C}_{p}^{(2)}(s)$ следующим образом. Объектом в $\mathcal{C}_{p}^{(2),+}(s)$ является или полупростой модуль, или такой модуль $\mathcal{N}$, что $\mathcal{N} / \mathcal{N}_{1}=\bigoplus_{j=1}^{m} x_{s}^{+}$для некоторого $m \in \mathbb{N}$, где $\mathcal{N}_{1}$ - максимальный полупростой подмодуль (цоколь); другими словами, объект в $\mathcal{C}_{p}^{(2),+}(s)$ - это модуль $\mathcal{J}_{\mathbf{X}^{+}}^{+}(m, n)$ при некоторых $m, n \in \mathbb{Z}_{+}$. Объекты в $\mathcal{C}_{p}^{(2),-}(s)$ определяются аналогично при $\mathcal{N} / \mathcal{N}_{1}=$ $\bigoplus_{j=1}^{m} \mathcal{X}_{p-s}^{-}$. Заметим, что $\mathrm{Ob}\left(\mathcal{C}_{p}^{(2)}(s)\right)=\mathrm{Ob}\left(\mathcal{C}_{p}^{(2),+}(s)\right) \cup \mathrm{Ob}\left(\mathfrak{C}_{p}^{(2),-}(s)\right)$.

\footnotetext{
${ }^{2)}$ Модули $\mathcal{W}_{s}^{+}(n), \mathcal{M}_{s}^{+}(n)$ и $\mathcal{O}_{s}^{+}(n, z)$ в 1.6 являются частными случаями модулей $\mathfrak{J}_{\mathbf{X}^{+}}^{+}(m, n)$.
} 
Теперь сведем классификацию модулей с полупростой длиной 2 к классификации неразложимых представлений колчана Кронекера $\mathbf{K}$. Читатель может обратиться к [13], [20] и к приложению Б по поводу необходимых фактов о колчанах.

2.5.2. Лемма. Каждая из категорий $\mathrm{C}_{p}^{(2),+}(s)$ и $\mathrm{C}_{p}^{(2),-}(s)$ эквивалентна категории $\operatorname{Rep}(\mathbf{K})$ представлений колчана Кронекера $\mathbf{K}$.

ДокАЗАТЕЛЬСтво. Лемма почти очевидна, если заметить, что два отображения $\varepsilon$ и $\bar{\varepsilon}$ в

$$
x_{p-s}^{-\stackrel{\bar{\varepsilon}}{\longrightarrow} \underset{\varepsilon}{\longrightarrow}} \mathcal{M}_{s}^{+}(2)
$$

составляют колчан в категории $\overline{\mathcal{U}}_{\mathfrak{q}} s \ell(2)$-представлений; применением функторов Hom в каждую из двух категорий устанавливается эквивалентность. Несколько более формально эквивалентность, например, между категориями $\mathcal{C}_{p}^{(2),+}(s)$ и $\operatorname{Rep}(\mathbf{K})$ дается функтором $\mathcal{F}$, который действует на объектах как

$$
\mathcal{F}\left(\mathcal{J}_{\mathbf{X}^{+}}^{+}(m, n)\right)=\left(\left(V_{0}, V_{1}\right),\left(r_{01}, \bar{r}_{01}\right)\right),
$$

где

$$
\begin{aligned}
& V_{0}=\operatorname{Hom}_{\overline{\mathcal{U}}_{\mathfrak{q}}}\left(\mathcal{M}_{s}^{+}(2), \mathcal{J}_{\mathbf{X}^{+}}^{+}(m, n)\right)=\mathbb{C}^{m}, \\
& V_{1}=\operatorname{Hom}_{\overline{\mathcal{U}}_{\mathfrak{q}}}\left(\mathcal{X}_{p-s}^{-}, \mathcal{J}_{\mathbf{X}^{+}}^{+}(m, n)\right)=\mathbb{C}^{n}
\end{aligned}
$$

и для двух линейно независимых гомоморфизмов $\varepsilon, \bar{\varepsilon} \in \operatorname{Hom}_{\overline{\mathcal{U}}_{\mathfrak{q}}}\left(\mathcal{X}_{p-s}^{-}, \mathcal{M}_{s}^{+}(2)\right)=\mathbb{C}^{2}$ два линейных отображения $r_{01}, \bar{r}_{01} \in \operatorname{Hom}_{\mathbb{C}}\left(V_{0}, V_{1}\right)$ определяются как

$$
r_{01}(\varphi)=\varphi \circ \varepsilon, \quad \bar{r}_{01}(\varphi)=\varphi \circ \bar{\varepsilon}
$$

для всех $\varphi \in V_{0}$; кроме того, имеется естественное действие на морфизмах.

Существование такого функтора $\mathcal{G}$, что и $\mathcal{G} \mathcal{F}$, и $\mathcal{F} \mathcal{G}$ являются тождественными функторами очевидным образом вытекает из определений категорий $\mathcal{C}_{p}^{(2),+}(s)$ и $\operatorname{Rep}(\mathbf{K})$.

Из 2.5.2 и Б.2.1 немедленно следует желаемая классификация конечномерных $\overline{\mathcal{U}}_{\mathfrak{q}} s \ell(2)$-модулей с полупростой длиной $\ell=2$, что и завершает доказательство 1.7 .

\section{3. ДОКАЗАТЕЛЬСТВО ТЕОРЕМЫ 1.8}

Схема доказательства такова. Сначала в п. 3.1 мы находим проективную и инъективную резольвенты неприводимых представлений. Далее в 3.2 вычисляется $\operatorname{Ext}_{\bar{u}_{q}}^{n}$ между неприводимыми представлениями в каждом $\mathcal{C}_{p}(s)$, а в 3.3 изучается алгебpa $\operatorname{Ext}^{\bullet}$.

3.1. Проективная и инъективная резольвенты неприводимых модулей. Непосредственное рассмотрение показывает, что отображения, определяемые в следующей лемме, составляют резольвенту. 
3.1.1. Лемма. Для каждого $1 \leqslant s \leqslant p-1$ модуль $X_{s}^{a}$ имеет проективную резольвенту

$$
\cdots \stackrel{\partial_{3}}{\longrightarrow} \mathcal{P}_{s}^{a} \oplus \mathcal{P}_{s}^{a} \oplus \mathcal{P}_{s}^{a} \stackrel{\partial_{2}}{\longrightarrow} \mathcal{P}_{p-s}^{-a} \oplus \mathcal{P}_{p-s}^{-a} \stackrel{\partial_{1}}{\longrightarrow} \mathcal{P}_{s}^{a} \stackrel{\partial_{0}}{\rightarrow} X_{s}^{a}
$$

где $n$-й член для четных $n$ имеет вид

$$
\stackrel{\partial_{n}}{\longrightarrow} \underbrace{\mathcal{P}_{p-s}^{-a} \oplus \cdots \oplus \mathcal{P}_{p-s}^{-a}}_{n} \stackrel{\partial_{n-1}}{\longrightarrow},
$$

граничный морфизм есть сквозное отображение в

$$
\partial_{n}: \underbrace{\mathcal{P}_{s}^{a} \oplus \cdots \oplus \mathcal{P}_{s}^{a}}_{n+1} \rightarrow \mathcal{W}_{s}^{a}(n+1) \longmapsto \underbrace{\mathcal{P}_{p-s}^{-a} \oplus \cdots \oplus \mathcal{P}_{p-s}^{-a}}_{n} ;
$$

$n$-й член и граничный морфизм $\partial_{n}$ для нечетных $n$ получаются заменой $а$ на $-a$ u $s$ нар $-s$.

Утверждение, двойственное 3.1.1, таково.

3.1.2. Лемма. Для каждого $s, 1 \leqslant s \leqslant p-1$, модуль $X_{s}^{a}$ имеет инъективную резольвенту

$$
X_{s}^{a} \stackrel{\delta_{0}}{\longmapsto} \mathcal{P}_{s}^{a} \stackrel{\delta_{1}}{\longrightarrow} \mathcal{P}_{p-s}^{-a} \oplus \mathcal{P}_{p-s}^{-a} \stackrel{\delta_{2}}{\longrightarrow} \mathcal{P}_{s}^{a} \oplus \mathcal{P}_{s}^{a} \oplus \mathcal{P}_{s}^{a} \stackrel{\delta_{3}}{\longrightarrow} \ldots
$$

где $n$-й член для четных $n$ имеет вид

$$
\stackrel{\delta_{n-1}}{\longrightarrow} \underbrace{\mathcal{P}_{p-s}^{-a} \oplus \cdots \oplus \mathcal{P}_{p-s}^{-a}}_{n} \stackrel{\delta_{n}}{\longrightarrow},
$$

кограничный морфизм есть сквозное отображение в

$$
\delta_{n}: \underbrace{\mathcal{P}_{p-s}^{-a} \oplus \cdots \oplus \mathcal{P}_{p-s}^{-a}}_{n} \rightarrow \mathcal{M}_{p-s}^{-a}(n+1) \longmapsto \underbrace{\mathcal{P}_{s}^{a} \oplus \cdots \oplus \mathcal{P}_{s}^{a}}_{n+1} ;
$$

$n$-й член и кограничный морфизм $\delta_{n}$ для нечетных $n$ получаются заменой $а$ на $-a$ u $s$ нар $-s$.

Вычислим теперь $\operatorname{Ext}_{\bar{u}_{\mathfrak{q}}}^{n}$ между неприводимыми модулями.

3.2. Лемма. При $1 \leqslant s \leqslant p-1$ и $a= \pm$ имеются изоморфизмы векторных пространств

$$
\begin{aligned}
& \operatorname{Ext}_{\overline{\mathcal{U}}_{\mathfrak{q}}}^{n}\left(X_{s}^{a}, X_{s}^{a}\right) \cong \begin{cases}\mathbb{C}^{n+1}, & \text { если п четно, } \\
0, & \text { если } \text { печетно, }\end{cases} \\
& \operatorname{Ext}_{\overline{\mathcal{U}}_{\mathfrak{q}}}^{n}\left(X_{s}^{a}, X_{p-s}^{-a}\right) \cong \begin{cases}0, & \text { если } \text { ч четно, } \\
\mathbb{C}^{n+1}, & \text { если } \text { печетно. }\end{cases}
\end{aligned}
$$


ДокАЗАтЕЛЬство. Применение контравариантного функтора $\operatorname{Hom}_{\overline{\mathcal{U}}_{\mathfrak{q}}}\left(-, X_{s}^{a}\right)$ к проективной резольвенте модуля $X_{s}^{a}$ дает коцепной комплекс

$$
\begin{aligned}
\mathbf{P}: 0 & \stackrel{\delta_{0}}{\longrightarrow} \operatorname{Hom}_{\overline{\mathcal{U}}_{\mathfrak{q}}}\left(\mathcal{P}_{s}^{a}, X_{s}^{a}\right) \stackrel{\delta_{1}}{\longrightarrow} \operatorname{Hom}_{\overline{\mathcal{U}}_{\mathfrak{q}}}\left(\mathcal{P}_{p-s}^{-a} \oplus \mathcal{P}_{p-s}^{-a}, X_{s}^{a}\right) \stackrel{\delta_{2}}{\longrightarrow} \\
& \stackrel{\delta_{2}}{\longrightarrow} \operatorname{Hom}_{\overline{\mathcal{U}}_{\mathfrak{q}}}\left(\mathcal{P}_{s}^{a} \oplus \mathcal{P}_{s}^{a} \oplus \mathcal{P}_{s}^{a}, X_{s}^{a}\right) \stackrel{\delta_{3}}{\longrightarrow} \cdots,
\end{aligned}
$$

где $(n+1)$-й член для четных $n$ имеет вид

$$
\stackrel{\delta_{n}}{\longrightarrow} \operatorname{Hom}_{\overline{\mathcal{U}}_{\mathfrak{q}}}(\underbrace{\mathcal{P}_{s}^{a} \oplus \cdots \oplus \mathcal{P}_{s}^{a}}_{n+1}, X_{s}^{a}) \cong \mathbb{C}^{n+1} \stackrel{\delta_{n+1}}{\longrightarrow},
$$

a $(n+1)$-й член для нечетных $n$ имеет вид

$$
\stackrel{\delta_{n}}{\longrightarrow} \operatorname{Hom}_{\overline{\mathcal{U}}_{q}}(\underbrace{\mathcal{P}_{p-s}^{-a} \oplus \cdots \oplus \mathcal{P}_{p-s}^{-a}}_{n+1}, X_{s}^{a}) \cong 0 \stackrel{\delta_{n+1}}{\longrightarrow} .
$$

Видим, что кограничные морфизмы $\delta_{i}=0, i \geqslant 1$. Но тогда

$$
\operatorname{Ext}_{\bar{u}_{\mathfrak{q}}}^{n}\left(X_{s}^{a}, X_{s}^{a}\right)=H^{n}(\mathbf{P})=\operatorname{ker}\left(\delta_{n+1}\right) / \operatorname{im}\left(\delta_{n}\right)= \begin{cases}\mathbb{C}^{n+1}, & \text { если } n \text { четно, } \\ 0, & \text { если } n \text { нечетно. }\end{cases}
$$

Для вычисления $\operatorname{Ext}_{\overline{\mathcal{U}}_{\mathfrak{q}}}^{n}\left(\mathcal{X}_{s}^{a}, \mathcal{X}_{p-s}^{-a}\right)$ применим функтор $\operatorname{Hom}_{\overline{\mathcal{U}}_{\mathfrak{q}}}\left(-, \mathcal{X}_{p-s}^{-a}\right)$ к $(3.1)$, и этим завершим доказательство.

Заметим далее, что квантовая группа Люстига действует на проективной резольвенте модуля $X_{s}^{a}$, и имеет место следующая лемма.

3.2.1. Лемма. Векторнъе пространства $\operatorname{Ext}_{\overline{\mathcal{u}}_{\mathfrak{q}}}^{n}\left(X_{s}^{a}, X_{s}^{a}\right)$ u $\operatorname{Ext}_{\overline{\mathcal{u}}_{\mathfrak{q}}}^{n}\left(X_{s}^{a}, X_{p-s}^{-a}\right)$ являются неприводимыми $е(2)$-модулями.

3.3. Ассоциативная алгебра Ext ${ }^{\bullet}$ Для каждой подкатегории $\mathcal{C}_{p}(s)$ при $1 \leqslant$ $s \leqslant p-1$ рассмотрим полную Ехt-группу

$$
\operatorname{Ext}^{\bullet} \equiv \operatorname{Ext}_{s}^{\bullet}=\bigoplus_{n \geqslant 0} \operatorname{Ext}_{\bar{u}_{\mathfrak{q}}}^{n}\left(X_{s}^{+} \oplus X_{p-s}^{-}, X_{s}^{+} \oplus X_{p-s}^{-}\right)
$$

В силу 3.2 имеется изоморфизм векторных пространств

$$
\operatorname{Ext}_{\bar{u}_{\mathfrak{q}}}^{n}\left(X_{s}^{+} \oplus X_{p-s}^{-}, X_{s}^{+} \oplus X_{p-s}^{-}\right) \cong \mathbb{C}^{n+1} \oplus \mathbb{C}^{n+1}, \quad n \in \mathbb{Z}_{+} .
$$

Будем писать Ext ${ }^{\bullet}$ вместо $\mathrm{Ext}_{s}^{\bullet}$, поскольку его алгебраическая структура в действительности не зависит от $s$. Градуированное векторное пространство Ext ${ }^{\bullet}$ снабжено структурой ассоциативной алгебры, определяемой произведением Йонеды.

Ниже, злоупотребляя обозначениями, будем писать просто представитель элемента из $\operatorname{Ext}_{\overline{\mathcal{u}}_{q}}^{n}$ вместо соответствующего класса эквивалентности.

Напомним, что базис $\left\{x_{i}^{+}\right\} \cup\left\{x_{i}^{-}\right\}, i=1,2$, в $\operatorname{Ext}_{\overline{\mathcal{u}}_{\mathfrak{q}}}\left(X_{s}^{+} \oplus \mathcal{X}_{p-s}^{-}, X_{s}^{+} \oplus \mathcal{X}_{p-s}^{-}\right) \cong \mathbb{C}^{2} \oplus \mathbb{C}^{2}$ можно выбрать так, что

$$
\begin{array}{ll}
x_{1}^{-}: X_{s}^{+} \longmapsto \mathcal{V}_{p-s}^{-} \rightarrow X_{p-s}^{-}, & x_{1}^{+}: X_{p-s}^{-} \longmapsto \mathcal{V}_{s}^{+} \rightarrow X_{s}^{+}, \\
x_{2}^{-}: X_{s}^{+} \longmapsto \overline{\mathcal{V}}_{p-s}^{-} \rightarrow X_{p-s}^{-}, & x_{2}^{+}: X_{p-s}^{-} \longmapsto \overline{\mathcal{V}}_{s}^{+} \rightarrow X_{s}^{+} .
\end{array}
$$


3.3.1. Лемма. Алгебра Ext ${ }^{\bullet}$ порождена элементами $x_{i}^{ \pm}, i=1,2$.

ДокаЗАтЕльство. Рассмотрим сначала $\operatorname{Ext}_{\overline{\mathcal{q}}_{\mathfrak{q}}}^{n}\left(X_{s}^{a}, X_{s}^{a}\right)$ для четных $n$. В силу 3.2 .1 пространство $\operatorname{Ext}_{\overline{\mathcal{u}}_{\mathfrak{q}}}^{n}\left(X_{s}^{a}, X_{s}^{a}\right)$ есть неприводимый $s \ell(2)$-модуль. Вектор старшего веса $x_{0}$ в этом модуле имеет вид

$$
x_{0}=\underbrace{x_{1}^{-a} \ldots x_{1}^{a} x_{1}^{-a} x_{1}^{a}}_{n} .
$$

Этот класс когомологий ненулевой, поскольку его ограничение на подалгебру $\mathbb{C}[F]$ в $\overline{\mathcal{U}}_{\mathfrak{q}} s \ell(2)$ ненулевое, в чем можно убедиться непосредственно. Ввиду $s \ell(2)$-действия, получаем, что $\operatorname{Ext}_{\overline{\mathcal{u}}_{\mathfrak{q}}}^{n}\left(X_{s}^{a}, X_{s}^{a}\right)$ порождается элементами $x_{i}^{ \pm}$. Для $\operatorname{Ext}_{\overline{\mathcal{u}}_{\mathfrak{q}}}^{n}\left(\mathcal{X}_{s}^{a}, X_{p-s}^{-a}\right)$ и нечетных $n$ рассуждение аналогично.

Взяв произведения Йонеды приведенных выше коротких точных последовательностей, получаем следующие точные последовательности в пространстве $\operatorname{Ext}_{\bar{u}_{\mathfrak{q}}}^{2}\left(X_{s}^{+}, X_{s}^{+}\right) \cong \mathbb{C}^{3}:$

$$
\begin{aligned}
& x_{1}^{-} x_{1}^{+}: x_{s}^{+} \longmapsto \mathcal{V}_{p-s}^{-} \rightarrow \mathcal{V}_{s}^{+} \rightarrow x_{s}^{+}, \\
& x_{2}^{-} x_{2}^{+}: x_{s}^{+} \longmapsto \overline{\mathcal{V}}_{p-s}^{-} \rightarrow \overline{\mathcal{V}}_{s}^{+} \rightarrow x_{s}^{+}, \\
& x_{2}^{-} x_{1}^{+}: x_{s}^{+} \longmapsto \overline{\mathcal{V}}_{p-s}^{-} \rightarrow \mathcal{V}_{s}^{+} \rightarrow x_{s}^{+}, \\
& x_{1}^{-} x_{2}^{+}: x_{s}^{+} \longmapsto \mathcal{V}_{p-s}^{-} \rightarrow \overline{\mathcal{V}}_{s}^{+} \rightarrow x_{s}^{+}
\end{aligned}
$$

и точные последовательности $x_{1}^{+} x_{1}^{-}, x_{2}^{+} x_{2}^{-}, x_{2}^{+} x_{1}^{-}, x_{1}^{+} x_{2}^{-}$в $\operatorname{Ext}_{\overline{\mathcal{u}}_{\mathfrak{q}}}^{2}\left(\mathcal{X}_{p-s}^{-}, x_{p-s}^{-}\right) \cong \mathbb{C}^{3}$.

3.3.2. Лемма. $x_{1}^{-} x_{2}^{+}+x_{2}^{-} x_{1}^{+}=x_{1}^{+} x_{2}^{-}+x_{2}^{+} x_{1}^{-}=0$.

ДокАЗАТЕЛЬСтво. Эти соотношения следуют из существования проективных модулей. Рассуждение для $x_{1}^{-} x_{2}^{+}+x_{2}^{-} x_{1}^{+}$можно развить следующим явным образом. Рассмотрим короткую точную последовательность

$$
X_{p-s}^{-} \oplus X_{p-s}^{-} \longmapsto \mathcal{M}_{s}^{+}(2) \rightarrow X_{s}^{+},
$$

которая дает длинную точную Ext-последовательность

$$
\begin{aligned}
& 0 \longrightarrow \operatorname{Hom}_{\overline{\mathcal{U}}_{\mathfrak{q}}}\left(X_{s}^{+}, X_{s}^{+}\right) \rightarrow \operatorname{Hom}_{\overline{\mathcal{U}}_{\mathfrak{q}}}\left(\mathcal{M}_{s}^{+}(2), X_{s}^{+}\right) \rightarrow \operatorname{Hom}_{\overline{\mathcal{U}}_{\mathfrak{q}}}\left(X_{p-s}^{-} \oplus X_{p-s}^{-}, X_{s}^{+}\right) \stackrel{\omega_{0}}{\longrightarrow} \\
& \stackrel{\omega_{0}}{\longrightarrow} \operatorname{Ext}_{\overline{\mathcal{U}}_{\mathfrak{q}}}\left(X_{s}^{+}, X_{s}^{+}\right) \rightarrow \operatorname{Ext}_{\overline{\mathcal{U}}_{\mathfrak{q}}}\left(\mathcal{M}_{s}^{+}(2), X_{s}^{+}\right) \stackrel{\varphi}{\longrightarrow} \operatorname{Ext}_{\overline{\mathcal{U}}_{\mathfrak{q}}}\left(X_{p-s}^{-} \oplus X_{p-s}^{-}, X_{s}^{+}\right) \stackrel{\omega_{1}}{\longrightarrow} \\
& \stackrel{\omega_{1}}{\longrightarrow} \operatorname{Ext}_{\overline{\mathcal{U}}_{\mathfrak{q}}}^{2}\left(X_{s}^{+}, X_{s}^{+}\right) \rightarrow \cdots,
\end{aligned}
$$

где $\omega_{0}$ и $\omega_{1}$ - связывающие гомоморфизмы, определяемые с точностью до знака произведением Йонеды на заданную короткую точную последовательность (3.3).

Рассмотрим короткую точную последовательность $y^{+} \in \operatorname{Ext} \frac{1}{\overline{\mathcal{q}}_{\mathfrak{q}}}\left(\mathcal{M}_{s}^{+}(2), \mathcal{X}_{s}^{+}\right)$, имеющую вид

$$
X_{s}^{+} \longmapsto \mathcal{P}_{s}^{+} \rightarrow \mathcal{M}_{s}^{+}(2) .
$$


Расширение $y^{+}$модуля $\mathcal{M}_{s}^{+}(2)$ определяет расширение подмодуля $\mathcal{X}_{p-s}^{-} \oplus \mathcal{X}_{p-s}^{-} \subset$ $\mathcal{M}_{s}^{+}(2)$ как точную последовательность $\varphi\left(y^{+}\right) \in \operatorname{Ext} \frac{1}{\bar{u}_{q}}\left(X_{p-s}^{-} \oplus \mathcal{X}_{p-s}^{-}, X_{s}^{+}\right)$, имеющую вид

$$
X_{s}^{+} \longmapsto \mathcal{W}_{p-s}^{-}(2) \rightarrow X_{p-s}^{-} \oplus X_{p-s}^{-} .
$$

Как следствие тождества $\omega_{1} \circ \varphi=0$ в $\operatorname{Ext}_{\overline{\mathcal{q}}_{\mathfrak{q}}}^{2}\left(X_{s}^{+}, X_{s}^{+}\right)$выполнено

$$
0=\omega_{1} \circ \varphi\left(y^{+}\right)=X_{s}^{+} \longmapsto \mathcal{W}_{p-s}^{-}(2) \rightarrow \mathcal{M}_{s}^{+}(2) \rightarrow X_{s}^{+}
$$

или, что эквивалентно,

$$
0=\omega_{1} \circ \varphi\left(y^{+}\right)=x_{1}^{-} x_{2}^{+}+x_{2}^{-} x_{1}^{+} .
$$

Равенство $x_{1}^{+} x_{2}^{-}+x_{2}^{+} x_{1}^{-}=0$ устанавливается аналогично с помощью короткой точной последовательности $X_{s}^{+} \oplus X_{s}^{+} \longmapsto \mathcal{M}_{p-s}^{-}(2) \rightarrow X_{p-s}^{-}$.

Из 3.3.1 и 3.3.2 следует, что алгебра Ext ${ }^{\bullet}$ порождена элементами $x_{i}^{ \pm}, i=1,2$, и имеет определяющие соотношения $x_{1}^{-} x_{2}^{+}+x_{2}^{-} x_{1}^{+}=0$ и $x_{1}^{+} x_{2}^{-}+x_{2}^{+} x_{1}^{-}=0$. Положим также $x_{i}^{+} x_{j}^{+}=x_{i}^{-} x_{j}^{-}=0, i, j=1,2$, поскольку произведение Йонеды определено только для двух точных последовательностей, конец одной из которых совпадает с началом другой. Этим завершается доказательство теоремы 1.8 .

\section{4. ЭКВИВАЛЕНТНОСТЬ КАТЕГОРИЙ ПРИ $P=2$ : ЯВНАЯ КОНСТРУКЦИЯ}

Докажем теперь эквивалентность категорий $\mathcal{W}(p)-$ и $\overline{\mathcal{U}}_{\mathfrak{q}} s \ell(2)$-представлений при $p=2$. Доказательство значительно упрощается по сравнению со случаем общих $p$, потому что $\mathcal{W}(p)$-генераторы явно строятся из симплектических фермионов [21]. Распространение доказательства на общие $p$ требует должного обобщения симплектических фермионов, достигаемого введением мультиплета конформных полей первого порядка, несущих $\overline{\mathcal{U}}_{q} s \ell(2)$-действие. Эта эквивалентность влечет, в частности, что приведенные в 1.6 диаграммы применимы в равной мере к неразложимым $\mathcal{W}(p)$ модулям.

4.1. $\mathcal{W}(2)$-алгебра и симплектические фермионы. При $p=2 \quad W$-алгебру $\mathcal{W}(2)$ [10], [22] (см. 1.2) удобно описывать в терминах так называемых симплектических фермионов [21] с операторным произведением (в голоморфном секторе, который мы и рассматриваем далее)

$$
\theta(z) \bar{\theta}(w)=\ln (z-w)
$$

Соответствующая алгебра Вирасоро с центральным зарядом $c=-2$ порождена тензором энергии-импульса (подразумевается нормальное упорядочение)

$$
T(z)=-\partial \theta(z) \partial \bar{\theta}(z)
$$

а алгебра $\mathcal{W}(2)$ порождена током $T(z)$ и двумя токами

$$
W^{+}(z)=\partial^{2} \theta(z) \partial \theta(z), \quad W^{-}(z)=\partial^{2} \bar{\theta}(z) \partial \bar{\theta}(z) .
$$


$(1,2)-$ Модель содержит два сектора полей: поля, порожденные токами $\theta(z)$ и $\bar{\theta}(z)$ из оператора 1 (периодические граничные условия) и из так называемого оператора твиста $\mu_{ \pm}(z)$ (антипериодические граничные условия); нижний индекс \pm указывает на две квантово-групповые компоненты. В секторе периодических граничных условий имеем

$$
\begin{aligned}
& \theta(z)=\theta_{0}^{*}+\theta_{0} \ln z-\sum_{n \neq 0} \frac{1}{n} \theta_{n} z^{-n}, \\
& \bar{\theta}(z)=\bar{\theta}_{0}^{*}+\bar{\theta}_{0} \ln z-\sum_{n \neq 0} \frac{1}{n} \bar{\theta}_{n} z^{-n},
\end{aligned}
$$

а в секторе антипериодических граничных условий -

$$
\begin{aligned}
& \theta(z)=\sum_{n \in \mathbb{Z}+1 / 2} \frac{1}{n} \theta_{n} z^{-n}, \\
& \bar{\theta}(z)=\sum_{n \in \mathbb{Z}+1 / 2} \frac{1}{n} \bar{\theta}_{n} z^{-n} .
\end{aligned}
$$

Коммутационные соотношения между модами в секторе периодических граничных условий имеют вид $([\cdot, \cdot]$ обозначает суперкоммутатор, а в данном случае - антикоммутатор)

$$
\left[\theta_{n}, \bar{\theta}_{m}\right]=n \delta_{m+n, 0}, \quad\left[\theta_{n}, \theta_{m}\right]=0, \quad\left[\bar{\theta}_{n}, \bar{\theta}_{m}\right]=0
$$

а единственные ненулевые коммутационные соотношения с участием $\theta_{0}^{*}$ и $\bar{\theta}_{0}^{*}$ суть ${ }^{3)}$

$$
\left[\theta_{0}, \bar{\theta}_{0}^{*}\right]=1, \quad\left[\bar{\theta}_{0}, \theta_{0}^{*}\right]=1 .
$$

4.2. Бозонизация. Другое описание модели (как в 1.2) достигается в терминах свободного бозонного поля, являющегося решением "уравнения Риккати"

$$
\frac{1}{2} \partial \varphi \partial \varphi(z)+\frac{1}{2} \partial^{2} \varphi(z)=T(z) .
$$

Это уравнение имеет два решения $\varphi^{+}(z)$ и $\varphi^{-}(z)$, являющиеся скалярными полями с операторными произведениями

$$
\varphi^{ \pm}(z) \varphi^{ \pm}(w)=\ln (z-w)
$$

и связанные равенством

$$
\varphi_{0}^{+}=-\varphi_{0}^{-}
$$

где

$$
\varphi_{0}^{ \pm}=\frac{1}{2 i \pi} \oint d z \partial \varphi^{ \pm}(z)
$$

3) Действительно, поскольку $\theta\left(z e^{2 i \pi}\right)=\theta(z)+2 i \pi \theta_{0}$, получаем, что антикоммутатор $\left[2 i \pi \theta_{0}, \bar{\theta}(w)\right]$ дается приращением операторного произведения $\theta(z) \bar{\theta}(w)$, когда $z$ продолжается посредством $z \rightarrow$ $w+(z-w) e^{2 i \pi}$, т.е. $\ln \left((z-w) e^{2 i \pi}\right)-\ln (z-w)=2 i \pi$. Это выражение не зависит от $w$, а потому $\left[\theta_{0}, \bar{\theta}_{0}^{*}\right]=1,\left[\theta_{0}, \bar{\theta}_{n}\right]=0$. 
Симплектические фермионы можно описывать в любой из двух взаимно нелокальных картин, т.е. либо в терминах $\varphi^{+}$, либо в терминах $\varphi^{-}$. В $\varphi^{+}$-картине имеем

$$
\partial \theta(z)=e^{-\varphi^{+}(z)}, \quad \bar{\theta}(z)=e^{\varphi^{+}(z)},
$$

а в $\varphi^{-}$-картине -

$$
\theta(z)=-e^{\varphi^{-}(z)}, \quad \partial \bar{\theta}(z)=e^{-\varphi^{-}(z)}
$$

Специальную роль играют нулевые моды

$$
\theta_{0}=\frac{1}{2 i \pi} \oint d u \partial \theta(u)=\frac{1}{2 i \pi} \oint d u e^{-\varphi^{+}(u)}
$$

и

$$
\bar{\theta}_{0}=\frac{1}{2 i \pi} \oint d u \partial \bar{\theta}(u)=\frac{1}{2 i \pi} \oint d u e^{-\varphi^{-}(u)} .
$$

В $\varphi^{+}$-картине $\theta_{0}$ является скрининг-оператором, а $\bar{\theta}_{0}-$ (нелокальным) оператором снятия контура; в $\varphi^{-}$-картине $\theta_{0}$ - оператор снятия контура, а $\bar{\theta}_{0}-$ скринингоператор.

В $\varphi^{-}$-картине конструкция из (1.1) воспроизводится выбором

$$
W^{-}(z)=e^{-2 \varphi^{-}(z)}=\partial^{2} \bar{\theta}(z) \partial \bar{\theta}(z), \quad S_{+}=\frac{1}{2 i \pi} \oint d u e^{2 \varphi^{-}(u)}=\frac{1}{2 i \pi} \oint d u \partial \theta(u) \theta(u) .
$$

Каждая из бозонизованных картин позволяет локально выразить компоненту оператора твиста. Начав для определенности с $\varphi^{+}$-картины, положим

$$
\mu_{+}(z)=e^{\varphi^{+}(z) / 2}
$$

Тогда вторая компонента имеет вид

$$
\mu_{-}(z)=\frac{1}{2 i \pi} \int_{C_{z}} d u e^{-\varphi^{+}(u)} e^{\varphi^{+}(z) / 2},
$$

где $C_{z}$ обозначает контур, проведенный из $-\infty \mathrm{k} z . \mathrm{B} \varphi^{-}$-картине при этом имеем

$$
\mu_{-}(z) \sim e^{\varphi^{-}(z) / 2} .
$$

Две компоненты являются векторами в двумерном представлении квантовой группы, см. ниже.

4.3. Квантовая группа. Как обычно, скрининг-оператор и оператор снятия контура порождают квантовую группу. В логарифмической $(1, p)$-модели конформной теории поля эта квантовая группа есть $\overline{\mathcal{U}}_{\mathfrak{q}} s \ell(2)$ при $\mathfrak{q}=e^{i \pi / p}$.

$\mathrm{B}(1,2)$-модели имеем $\mathfrak{q}=i$, а генераторы квантовой группы явно строятся как

$$
E=\theta_{0}, \quad F=\pi \bar{\theta}_{0} K
$$

(причем $\left.E^{2}=F^{2}=0\right)$, где

$$
K=e^{ \pm i \pi \varphi_{0}^{ \pm}}=e^{-\frac{1}{2} \oint \partial \theta \bar{\theta}}=e^{\frac{1}{2} \oint \theta \partial \bar{\theta}} .
$$

Нулевые моды $\theta_{0}^{*}$ и $\bar{\theta}_{0}^{*}$ можно при этом рассматривать как функции на квантовой группе. Имеем также

$$
\theta_{0} \bar{\theta}_{0}+\bar{\theta}_{0} \theta_{0}=\frac{\mathbf{1}-K^{2}}{2 i \pi}
$$


4.4. $\mathcal{W}(2)$ - и $\overline{\mathcal{U}}_{\mathfrak{q}} s \ell(2)$-представления. Напомним [22], [23], [12], что алгебра $\mathcal{W}(2)$ имеет четыре неприводимых представления $\mathfrak{X}_{1}^{ \pm}$и $\mathfrak{X}_{2}^{ \pm}$. Представления $\mathfrak{X}_{2}^{ \pm}$являются модулями Стейнберга (неприводимыми модулями, модулями Верма и проективными модулями одновременно). Нам понадобятся также проективные модули $\mathfrak{P}_{1}^{ \pm}$, структура подфакторов которых описывается как

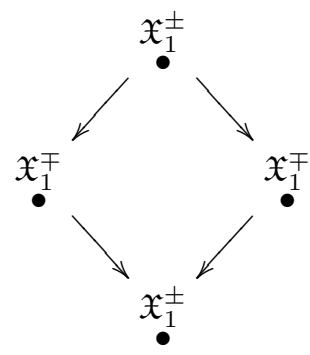

Поле, соответствующее вектору старшего веса в модуле $\mathfrak{P}_{1}^{+}$, есть $\mathbb{L}(z)=\theta(z) \bar{\theta}(z)$ (“логарифмический партнер" оператора 1).

Чтобы построить $\mathcal{W}(2)$-представление в терминах симплектических фермионов, введем вакуумный вектор $|0\rangle$, соответствующий оператору 1 (на который симплектические фермионы действуют целочисленными модами, см. (4.1)); он эквивалентным образом определяется условиями старшего веса

$$
\begin{aligned}
\theta_{n}|0\rangle & =0, \quad n \in \mathbb{Z}_{\geqslant 0}, \\
\bar{\theta}_{n}|0\rangle & =0, \quad n \in \mathbb{Z}_{\geqslant 0}, \\
K|0\rangle & =|0\rangle,
\end{aligned}
$$

где мы одновременно указали действие генератора $K(4.7)$.

Пусть $M$ - модуль, порожденный из $|0\rangle$ целочисленными модами $\theta_{n \leqslant-1}$ и $\bar{\theta}_{n \leqslant-1}$. "Целочисленный” модуль над симплектическими фермионами имеет тогда вид

$$
\boldsymbol{M}=M+\theta_{0}^{*} M+\bar{\theta}_{0}^{*} M+\bar{\theta}_{0}^{*} \theta_{0}^{*} M .
$$

Как $\mathcal{W}(2)$-модуль $\boldsymbol{M}$ разлагается в прямую сумму двух проективных модулей: модуля $\mathfrak{P}_{1}^{+}$, порожденного элементом $\theta_{0}^{*} \bar{\theta}_{0}^{*}|0\rangle$, и модуля $\mathfrak{P}_{1}^{-}$, порожденного элементом $\theta_{-1} \theta_{0}^{*} \bar{\theta}_{0}^{*}|0\rangle$.

Как $\overline{\mathcal{U}}_{\mathfrak{q}} s \ell(2)$-модуль $\boldsymbol{M}$ разлагается в бесконечную сумму проективных модулей. Для любого состояния $|v\rangle=v|0\rangle \in M$, где $v$ - однородный многочлен четного порядка по $\theta_{\leqslant-1}$ и $\bar{\theta} \leqslant-1$, проективный $\overline{\mathcal{U}}_{\mathfrak{q}} s \ell(2)$-модуль $\mathcal{P}_{1}^{+}$(см. (1.2)) линейно порожден элементами $\left\{\bar{\theta}_{0}^{*} \theta_{0}^{*}|v\rangle, \theta_{0}^{*}|v\rangle,-\bar{\theta}_{0}^{*}|v\rangle,|v\rangle\right\}$. Аналогично для любого состояния $|v\rangle=v|0\rangle \in M$, где $v$ - однородный многочлен нечетного порядка по $\theta \leqslant-1$ и $\bar{\theta} \leqslant-1$, проективный $\overline{\mathcal{U}}_{\mathfrak{q}} s \ell(2)$-модуль $\mathcal{P}_{1}^{-}$линейно порожден элементами $\left\{\bar{\theta}_{0}^{*} \theta_{0}^{*}|v\rangle\right.$, $\left.\theta_{0}^{*}|v\rangle,-\bar{\theta}_{0}^{*}|v\rangle,|v\rangle\right\}$.

Далее, пусть $\left|\mu_{ \pm}\right\rangle$- вектор, соответствующий оператору $\mu_{ \pm}$, на который симплектические фермионы действуют полуцелыми модами, см. (4.2). Он эквивалентным 
образом определяется условиями старшего веса

$$
\begin{array}{ll}
\theta_{n}\left|\mu_{ \pm}\right\rangle=0, & n \in \frac{1}{2}+\mathbb{Z}_{\geqslant 0}, \\
\bar{\theta}_{n}\left|\mu_{ \pm}\right\rangle=0, & n \in \frac{1}{2}+\mathbb{Z}_{\geqslant 0}, \\
K\left|\mu_{ \pm}\right\rangle= \pm i\left|\mu_{ \pm}\right\rangle . &
\end{array}
$$

Кроме того, он удовлетворяет соотношениям

$$
\theta_{0}\left|\mu_{-}\right\rangle=\bar{\theta}_{0}\left|\mu_{+}\right\rangle=0 .
$$

Векторы $\left|\mu_{ \pm}\right\rangle$являются двумя базисными векторами в двумерном представлении алгебры $\overline{\mathcal{U}}_{\mathfrak{q}} s \ell(2)$, причем скрининг-операторы $\theta_{0}$ и $\bar{\theta}_{0}$ действуют, как указано в (4.5), а именно,

$$
\theta_{0}\left|\mu_{+}\right\rangle=\left|\mu_{-}\right\rangle, \quad \bar{\theta}_{0}\left|\mu_{-}\right\rangle=\frac{i}{\pi}\left|\mu_{+}\right\rangle .
$$

Пусть $\boldsymbol{N}_{ \pm}$обозначает модуль над симплектическими фермионами, порожденный из $\left|\mu_{ \pm}\right\rangle$полуцелыми модами.

Как $\mathcal{W}(2)$-модуль каждый из модулей $\boldsymbol{N}_{ \pm}$разлагается в два стейнберговских $\mathcal{W}(2)$-модуля $\mathfrak{X}_{2}^{+}$и $\mathfrak{X}_{2}^{-}$.

Как $\overline{\mathcal{U}}_{\mathfrak{q}} s \ell(2)$-модуль прямая сумма $\mathcal{W}(2)$-модулей $\boldsymbol{N}_{+} \oplus \boldsymbol{N}_{-}$разлагается в бесконечную сумму стейнберговских модулей. Стейнберговский модуль $x_{2}^{+}$линейно порожден элементами $\left\{v\left|\mu_{+}\right\rangle, v\left|\mu_{-}\right\rangle\right\}$для всякого однородного многочлена $v$ четного порядка по $\theta_{\leqslant-1}$ и $\bar{\theta}_{\leqslant-1}$; аналогично стейнберговский модуль $X_{2}^{-}$линейно порожден элементами $\left\{v\left|\mu_{+}\right\rangle, v\left|\mu_{-}\right\rangle\right\}$для всякого однородного многочлена $v$ нечетного порядка по $\theta \leqslant-1 / 2$ и $\bar{\theta}_{\leqslant-1 / 2}$.

4.5. Эквивалентность Мориты и доказательство теоремы 1.4. Пусть $\mathbb{M}$ обозначает модуль

$$
\mathbb{M}=M \oplus N_{+} \oplus N_{-} .
$$

Как $\mathcal{W}(2)$-модуль он разлагается в сумму всех проективных модулей $\mathfrak{P}_{1}^{+}, \mathfrak{P}_{1}^{-}$, $\mathfrak{X}_{2}^{+}$и $\mathfrak{X}_{2}^{-}$с кратностями, задаваемыми размерностями соответствующих квантовогрупповых модулей:

$$
\mathbb{M}=\mathfrak{P}_{1}^{+} \otimes \mathfrak{X}_{1}^{+} \oplus \mathfrak{P}_{1}^{-} \otimes \mathcal{X}_{1}^{-} \oplus \mathfrak{X}_{2}^{+} \otimes \mathfrak{X}_{2}^{+} \oplus \mathfrak{X}_{2}^{-} \otimes X_{2}^{-} .
$$

Kaк $\overline{\mathcal{U}}_{\mathfrak{q}} s \ell(2)$-модуль $\mathbb{M}$ также разлагается в сумму всех проективных модулей, с "кратностями", даваемыми $\mathcal{W}(2)$-модулями:

$$
\mathbb{M}=\mathfrak{X}_{1}^{+} \otimes \mathcal{P}_{1}^{+} \oplus \mathfrak{X}_{1}^{-} \otimes \mathcal{P}_{1}^{-} \oplus \mathfrak{X}_{2}^{+} \otimes \mathfrak{X}_{2}^{+} \oplus \mathfrak{X}_{2}^{-} \otimes \mathfrak{X}_{2}^{-} .
$$

Тот факт, что $\mathbb{M}$ является одновременно и $\mathcal{W}(2)$-модулем, и $\overline{\mathcal{U}}_{\mathfrak{q}} s \ell(2)$-модулем и разлагается по проективным модулям в каждом случае, позволяет стандартным образом установить эквивалентность категорий представлений. Построим прежде всего функтор $\overline{\mathcal{F}}: \mathfrak{C}_{2} \rightarrow \mathfrak{C}_{2}$. Удобно сначала определить контравариантный функтор

$$
\widetilde{\mathcal{F}}(y)=\operatorname{Hom}_{\overline{\mathcal{U}}_{\mathfrak{q}} s \ell(2)}(\mathbb{M}, y),
$$


который дает $\mathcal{W}(2)$-модуль для всякого $\overline{\mathcal{U}}_{\mathfrak{q}} s \ell(2)$-модуля $y$, а далее взять его композицию с функтором $\mathbf{C}: \mathfrak{C}_{2} \rightarrow \mathfrak{C}_{2}$, который заменяет каждый $\mathcal{W}(2)$-модуль на его контрагредиентный. Таким образом, $\overline{\mathcal{F}}=\mathbf{C} \widetilde{\mathcal{F}}: \mathfrak{C}_{2} \rightarrow \mathfrak{C}_{2}$ является ковариантным функтором.

Аналогично построим функтор $\mathcal{F}: \mathfrak{C}_{2} \rightarrow \mathfrak{C}_{2}$. Для любого $\mathcal{W}(2)$-модуля $\mathfrak{X}$ полагаem

$$
\mathcal{F}(\mathfrak{X})=\mathcal{C} \operatorname{Hom}_{\mathcal{W}(2)}(\mathbb{M}, \mathfrak{X})
$$

(что является $\overline{\mathcal{U}}_{\mathfrak{q}} s \ell(2)$-модулем), где $\mathcal{C}: \mathcal{C}_{2} \rightarrow \mathcal{C}_{2}-$ функтор взятия контрагредиентного модуля.

Функторы $\mathcal{F}$ и $\overline{\mathcal{F}}$ отправляют неприводимые представления в неприводимые, откуда немедленно следует, что

$$
\overline{\mathcal{F}} \overline{\mathcal{F}} \sim \operatorname{Id}_{\mathfrak{C}_{2}}, \quad \overline{\mathcal{F}} \mathcal{F} \sim \operatorname{Id}_{\mathfrak{C}_{2}}
$$

Естественная структура сплетения в конформной теории поля, определяемая манипуляциями с контурами в комплексной плоскости, совпадает со сплетением, определяемым квантово-групповой $R$-матрицей. Это иллюстрируется ниже на нескольких характерных примерах. Заметим сперва, что, как уже отмечалось во введении, квантовая группа $\overline{\mathcal{U}}_{\mathfrak{q}} s \ell(2)$ при $\mathfrak{q}=e^{i \pi / p}$ не является квазитреугольной, но является подалгеброй в большей квантовой группе, порожденной элементами $E, F$ и $k$ такими, что $k^{2}=K$, которая является квазитреугольной [11]. При $p=2$ универсальная $R$-матрица для этой большей квантовой группы принимает вид

$$
R=\frac{1}{8} \sum_{n, m=0}^{7}\left(i^{-n m / 2}+2 i^{n-m-n m / 2+1} E \otimes F\right) k^{n} \otimes k^{m},
$$

где выбрано, что $k=e^{+i \pi \varphi_{0}^{+} / 2}$ соответствует матрице сплетения $B^{+}$.

Ввиду справедливости равенств (4.6) определено действие $R$ на вертексные операторы, что позволяет отождествить $R$ с $B^{+}$-матрицей. Рассматриваемую в этом качестве $R$-матрицу можно переписать в виде

$$
R=\frac{1}{8} \sum_{n, m=0}^{7}\left(i^{-n m / 2}+2 \pi i^{n-m-n m / 2+1} \theta_{0} \otimes \bar{\theta}_{0} e^{i \pi \varphi_{0}^{+}}\right) e^{i \pi n \varphi_{0}^{+} / 2} \otimes e^{i \pi m \varphi_{0}^{+} / 2} .
$$

Отсюда получаем соотношения сплетения

$$
\begin{aligned}
A(z) B(w)=R B(w) A(z)=\frac{1}{8} \sum_{n, m=0}^{7} i^{-n m / 2} e^{i \pi n \varphi_{0}^{+} / 2} B(w) e^{i \pi m \varphi_{0}^{+} / 2} A(z)- \\
-\frac{1}{8} \sum_{n, m=0}^{7} 2 \pi i^{-n m / 2+1} e^{i \pi n \varphi_{0}^{+} / 2} \theta_{0} B(w) e^{i \pi(m+2) \varphi_{0}^{+} / 2} \bar{\theta}_{0} A(z)
\end{aligned}
$$

для любых двух полей $A(z)$ и $B(z)$, где $z$ и $w$ являются вещественными и $z>w$ (соотношения затем аналитически продолжаются с вещественной оси). 
В частности, имеем

$$
\theta(z) \bar{\theta}(w)=-\bar{\theta}(w) \theta(z)-2 i \pi, \quad \bar{\theta}(z) \theta(w)=-\theta(w) \bar{\theta}(z), \quad z>w .
$$

Эти соотношения можно проиллюстрировать процедурой перестановки операторов в комплексной плоскости. В первом соотношении в (4.11) в $\varphi^{+}$-картине (см. (4.3)), оператор $\theta(z)$ является нелокальным, $\theta(z)=\int^{z} d u e^{-\varphi^{+}(u)}$, что можно представить контуром интегрирования из бесконечности в точку, где находится оператор:

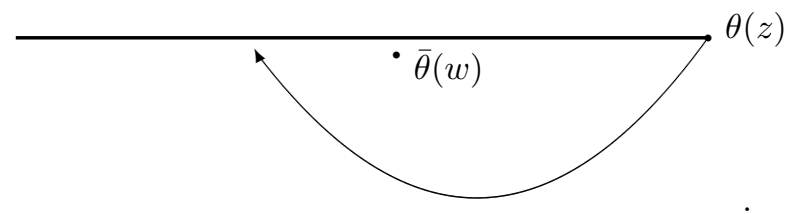

Обнесение его по полуокружности (по часовой стрелке) вокруг $w$ изменяет контур интегрирования, что приводит к появлению дополнительного слагаемого, равного $-2 i \pi$. Такого слагаемого не возникает во втором соотношении в (4.11), что можно представить как

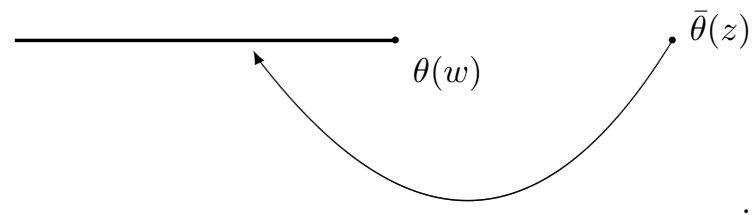

Аналогично для поля $\mathbb{L}(z)=\theta(z) \bar{\theta}(z)$, которое является логарифмическим партнером оператора $\mathbf{1}$, из (4.10) следует, что

$$
\mathbb{L}(z) \mathbb{L}(w)=\mathbb{L}(w) \mathbb{L}(z)-2 i \pi \theta(w) \bar{\theta}(z),
$$

а для оператора твиста имеем

$$
\begin{aligned}
& \mu_{ \pm}(z) \mu_{ \pm}(w)=e^{i \pi / 4} \mu_{ \pm}(w) \mu_{ \pm}(z), \quad \mu_{+}(z) \mu_{-}(w)=e^{i \pi / 4} \mu_{-}(w) \mu_{+}(z) \\
& \mu_{-}(z) \mu_{+}(w)=e^{i \pi / 4}\left(\mu_{+}(w) \mu_{-}(z)+2 i \mu_{-}(w) \mu_{+}(z)\right) .
\end{aligned}
$$

Ленточная структура $\overline{\mathcal{U}}_{\mathfrak{q}} s \ell(2)$ задается центральным элементом

$$
\boldsymbol{v}=\frac{1-i}{2 \sqrt{2}}\left(\left(e^{i \pi / 4}-2 e^{-i \pi / 4} K F E\right)\left(\mathbf{1}+K^{2}\right)+(K+2 i F E)\left(\mathbf{1}-K^{2}\right)\right) .
$$

С учетом (4.6) получаем элемент

$$
\begin{aligned}
\boldsymbol{v}= & \frac{1-i}{2 \sqrt{2}}\left(\left(e^{i \pi / 4}+2 \pi e^{i \pi\left(2 \varphi_{0}^{+}-1 / 4\right)} \bar{\theta}_{0} \theta_{0}\right)\left(\mathbf{1}+e^{2 i \pi \varphi_{0}^{+}}\right)+\right. \\
& \left.+2\left(\mathbf{1}-2 i \pi \bar{\theta}_{0} \theta_{0}\right) e^{i \pi \varphi_{0}^{+}}\left(\mathbf{1}-e^{2 i \pi \varphi_{0}^{+}}\right)\right)
\end{aligned}
$$

который действует на пространстве состояний как

$$
\boldsymbol{v}=e^{2 i \pi L_{0}} .
$$




\section{5. ЗАКЛЮЧЕНИЕ}

В силу эквивалентности Каждана-Люстига структура неразложимых модулей, изображенных в 1.6, одновременно описывает структуру неразложимых $\mathcal{W}(p)$-модулей. Проективная резольвента (3.1) также применима к алгебре $\mathcal{W}(p)$, и ее можно поэтому использовать в конформной теории поля подобно известным резольвентам (фельдеровского типа) в рациональных конформных теориях поля.

В качестве применения найденной в 1.8 алгебры Ext ${ }^{\bullet}$ заметим следующее. Для подкатегории $\mathcal{C}_{p}(s)$ в категории $\mathcal{C}_{p}$ (рассматриваемой либо как категория $\mathcal{W}(p)$ представлений, либо как категория $\overline{\mathcal{U}}_{\mathfrak{q}} s \ell(2)$-представлений $)$, ее производная категория эквивалентна производной категории когерентных пучков на некотором некоммутативном расширении $\mathbb{C P}^{1}[24]$. Алгебра Ext ${ }^{\bullet}$ является координатным кольцом этого некоммутативного расширения.

Кроме установления эквивалентности категорий, построенный в (4.9) бимодуль $\mathbb{M}$ имеет и другое применение: этот бимодуль можно использовать при построении полной конформной теории поля [3], [4], [25]. Имея $\mathbb{M}$ (модуль над квантовой группой и над алгеброй $\mathcal{W}(p)$ в голоморфном секторе) и его второй экземпляр $\overline{\mathbb{M}}$, где действие алгебры $\mathcal{W}(p)$ заменено ее действием в антиголоморфном секторе, можно стандартным образом построить полную конформную теорию путем взятия тензорного произведения $\mathbb{M} \otimes_{\mathfrak{G}} \overline{\mathbb{M}}$ над квантовой группой $\mathfrak{G}=\overline{\mathcal{U}}_{\mathfrak{q}} s \ell(2)$. Другие возможности состоят в том, чтобы взять $\mathbb{M} \otimes_{\mathfrak{G}} N \otimes_{\mathfrak{G}} \overline{\mathbb{M}}$, где $N$ - любой $\mathfrak{G}$-бимодуль с ассоциативным произведением $N \otimes N \rightarrow N$, согласованным со структурой бимодуля. Бимодуль $\mathbb{M}$ можно также использовать для получения пространства, двойственного к центру квантовой группы, - пространства "расширенных характеров" в логарифмических конформных моделях. "Расширенные характеры" можно получать взятием следа оператора $e^{2 i \pi \tau L_{0}}$ по бимодулю $\mathbb{M}$ (по компоненте, отвечающей вертекс-операторной алгебре), что должно давать версию общей конструкции из [7].

ПРИЛОЖЕНИЕ А

$$
\overline{\mathcal{U}}_{\mathfrak{q}} s \ell(2)-\text { модули }
$$

А.1. Модули $\mathcal{W}_{s}^{ \pm}(2), \mathcal{M}_{s}^{ \pm}(2)$ и $\mathcal{O}_{s}^{ \pm}(1, z)$.

А.1.1. Модуль $\mathcal{W}_{s}^{ \pm}(2)$. Пусть $s$ - целое, $1 \leqslant s \leqslant p-1$, и $a= \pm$. Модуль $\mathcal{W}_{s}^{a}(2)$ (см. 1.6) имеет три подфактора: $X_{s}^{a}$ ("левый"), $X_{p-s}^{-a}$ ("нижний") и $X_{s}^{a}$ ("правый"). Базис можно выбрать как

$$
\left\{\mathrm{a}_{n}, \mathrm{~b}_{n}\right\}_{0 \leqslant n \leqslant s-1} \cup\left\{\mathrm{x}_{k}\right\}_{0 \leqslant k \leqslant p-s-1},
$$

где $\left\{\mathrm{a}_{n}\right\}_{0 \leqslant n \leqslant s-1}$ - базис, соответствующий правому модулю $X_{s}^{a}, \quad\left\{\mathrm{~b}_{n}\right\}_{0 \leqslant n} \leqslant s-1$ - левому модулю $X_{s}^{a}$, а $\left\{\mathrm{x}_{k}\right\}_{0 \leqslant k \leqslant p-s-1}$ - нижнему модулю $X_{p-s}^{-a}$, причем $\overline{\mathcal{U}}_{\mathfrak{q}} s \ell(2)$ - 
действие задается формулами

$$
\begin{aligned}
K \mathrm{a}_{n} & =a q^{s-1-2 n} \mathrm{a}_{n}, \quad K \mathrm{~b}_{n}=a q^{s-1-2 n} \mathrm{~b}_{n}, \quad 0 \leqslant n \leqslant s-1, \\
K \mathrm{x}_{k} & =-a q^{p-s-1-2 k} \mathrm{x}_{k}, \quad 0 \leqslant k \leqslant p-s-1, \\
E \mathrm{x}_{k} & =-a[k][p-s-k] \mathrm{x}_{k-1}, \quad 0 \leqslant k \leqslant p-s-1 \quad\left(\mathrm{x}_{-1} \equiv 0\right), \\
E \mathrm{a}_{n} & = \begin{cases}a[n][s-n] \mathrm{a}_{n-1}, & 1 \leqslant n \leqslant s-1, \\
\mathrm{x}_{p-s-1}, & n=0,\end{cases} \\
E \mathrm{~b}_{n} & =a[n][s-n] \mathrm{b}_{n-1}, \quad 0 \leqslant n \leqslant s-1 \quad\left(\mathrm{~b}_{-1} \equiv 0\right),
\end{aligned}
$$

и

$$
\begin{aligned}
F \mathrm{a}_{n} & =\mathrm{a}_{n+1}, \quad 0 \leqslant n \leqslant s-1 \quad\left(\mathrm{a}_{s} \equiv 0\right), \\
F \mathrm{~b}_{n} & = \begin{cases}\mathrm{b}_{n+1}, & 0 \leqslant n \leqslant s-2, \\
\mathrm{x}_{0}, & n=s-1,\end{cases} \\
F \mathrm{x}_{k} & =\mathrm{x}_{k+1}, \quad 0 \leqslant k \leqslant p-s-1 \quad\left(\mathrm{x}_{p-s} \equiv 0\right) .
\end{aligned}
$$

А.1.2. Модуль $\mathcal{M}_{s}^{ \pm}(2)$. Пусть $s$ - целое, $1 \leqslant s \leqslant p-1$, и $a= \pm$. Модуль $\mathcal{M}_{s}^{a}(2)$, являющийся расширением

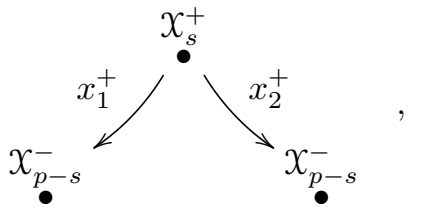

имеет базис

$$
\left\{\mathrm{a}_{n}\right\}_{0 \leqslant n \leqslant s-1} \cup\left\{\mathrm{x}_{k}, \mathrm{y}_{k}\right\}_{0 \leqslant k \leqslant p-s-1},
$$

где базис $\left\{\mathrm{a}_{n}\right\}_{0 \leqslant n \leqslant s-1}$ соответствует верхнему модулю $X_{s}^{a}$ в (A.1), базис $\left\{\mathrm{x}_{k}\right\}_{0 \leqslant k \leqslant p-s-1}-$ левому $X_{p-s}^{-a}$, а базис $\left\{\mathrm{y}_{k}\right\}_{0 \leqslant k \leqslant p-s-1}-$ правому $X_{p-s}^{-a}$, причем $\overline{\mathcal{U}}_{\mathfrak{q}} s \ell(2)$-действие задается формулами

$$
\begin{aligned}
& K \mathrm{a}_{n}=a q^{s-1-2 n} \mathrm{a}_{n}, \quad 0 \leqslant n \leqslant s-1, \\
& K \mathrm{x}_{k}=-a q^{p-s-1-2 k} \mathrm{x}_{k}, \quad K \mathrm{y}_{k}=-a q^{p-s-1-2 k} \mathrm{y}_{k}, \quad 0 \leqslant k \leqslant p-s-1, \\
& E \mathrm{x}_{k}=-a[k][p-s-k] \mathrm{x}_{k-1}, \\
& E \mathrm{y}_{k}=-a[k][p-s-k] \mathrm{y}_{k-1}, \quad 0 \leqslant k \leqslant p-s-1 \quad\left(\mathrm{x}_{-1} \equiv \mathrm{y}_{-1} \equiv 0\right), \\
& E \mathrm{a}_{n}= \begin{cases}a[n][s-n] \mathrm{a}_{n-1}, & 1 \leqslant n \leqslant s-1, \\
\mathrm{x}_{p-s-1}, & n=0,\end{cases}
\end{aligned}
$$

и

$$
\begin{gathered}
F \mathrm{x}_{k}=\mathrm{x}_{k+1}, \quad F \mathrm{y}_{k}=\mathrm{y}_{k+1}, \quad 0 \leqslant k \leqslant p-s-1 \quad\left(\mathrm{x}_{p-s} \equiv \mathrm{y}_{p-s} \equiv 0\right), \\
F \mathrm{a}_{n}= \begin{cases}\mathrm{a}_{n+1}, & 0 \leqslant n \leqslant s-2, \\
\mathrm{y}_{0}, & n=s-1 .\end{cases}
\end{gathered}
$$


А.1.3. Модуль $\mathcal{O}_{s}^{ \pm}(1, z)$. Пусть $s$ - целое, $1 \leqslant s \leqslant p-1, a= \pm$ и $z=z_{1}: z_{2}$. При $n=1$ диаграмма $\mathcal{O}_{s}^{a}(1, z)$ модуля из 1.6 принимает вид

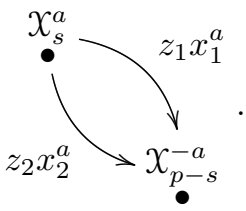

В терминах соответствующих базисов $\left\{\mathrm{a}_{n}\right\}_{0 \leqslant n \leqslant s-1}$ и $\left\{\mathrm{x}_{k}\right\}_{0 \leqslant k \leqslant p-s-1}$ в $X_{s}^{a}$ и $X_{p-s}^{-a}$, модуль $\mathcal{O}_{s}^{a}(1, z)$ имеет базис

$$
\left\{\mathrm{a}_{n}\right\}_{0 \leqslant n \leqslant s-1} \cup\left\{\mathrm{x}_{k}\right\}_{0 \leqslant k \leqslant p-s-1},
$$

причем $\overline{\mathcal{U}}_{\mathfrak{q}} s \ell(2)$-действие задается формулами

$$
\begin{aligned}
& K \mathrm{a}_{n}=a q^{s-1-2 n} \mathrm{a}_{n}, \quad K \mathrm{x}_{k}=-a q^{p-s-1-2 k} \mathrm{x}_{k}, \quad 0 \leqslant n \leqslant s-1, \quad 0 \leqslant k \leqslant p-s-1, \\
& E \mathrm{a}_{n}= \begin{cases}a[n][s-n] \mathrm{a}_{n-1}, & 1 \leqslant n \leqslant s-1, \\
z_{2} \mathrm{x}_{p-s-1}, & n=0,\end{cases} \\
& E \times_{k}=-a[k][p-s-k] \times_{k-1}, \quad 0 \leqslant k \leqslant p-s-1,
\end{aligned}
$$

где мы полагаем $\mathrm{x}_{-1}=0$, и

$$
\begin{gathered}
F \mathrm{a}_{n}= \begin{cases}\mathrm{a}_{n+1}, & 0 \leqslant n \leqslant s-2, \\
z_{1} \mathrm{x}_{0}, & n=s-1,\end{cases} \\
F \mathrm{x}_{k}=\mathrm{x}_{k+1}, \quad 0 \leqslant k \leqslant p-s-1,
\end{gathered}
$$

где мы полагаем $\mathrm{x}_{p-s}=0$. Тот факт, что тем самым определяется $\overline{\mathcal{U}}_{\mathfrak{q}} s \ell(2)$-модуль, проверяется прямым вычислением.

А.2. Проективные модули $\mathcal{P}_{s}^{ \pm}$. Пусть $s$ - целое, $1 \leqslant s \leqslant p-1$, и $a= \pm$. Проективный модуль $\mathcal{P}_{s}^{a}$ имеет базис

$$
\left\{\mathrm{a}_{n}, \mathrm{~b}_{n}\right\}_{0 \leqslant n \leqslant s-1} \cup\left\{\mathrm{x}_{k}, \mathrm{y}_{k}\right\}_{0 \leqslant k \leqslant p-s-1},
$$

где базис $\left\{\mathrm{b}_{n}\right\}_{0 \leqslant n \leqslant s-1}$ соответствует верхнему модулю $X_{s}^{a}$ в (1.2), базис $\left\{\mathrm{a}_{n}\right\}_{0 \leqslant n \leqslant s-1}-$ нижнему $X_{s}^{a}$, базис $\left\{\mathrm{x}_{k}\right\}_{0 \leqslant k \leqslant p-s-1}-$ правому $X_{p-s}^{-a}$, а базис $\left\{\mathrm{y}_{k}\right\}_{0 \leqslant k \leqslant p-s-1}-$ левому модулю $X_{p-s}^{-a}$, причем $\overline{\mathcal{U}}_{\mathfrak{q}} s \ell(2)$-действие задается как

$$
\begin{gathered}
K \mathrm{x}_{k}=-a q^{p-s-1-2 k} \mathrm{x}_{k}, \quad K \mathrm{y}_{k}=-a q^{p-s-1-2 k} \mathrm{y}_{k}, \quad 0 \leqslant k \leqslant p-s-1, \\
K \mathrm{a}_{n}=a q^{s-1-2 n} \mathrm{a}_{n}, \quad K \mathrm{~b}_{n}=a q^{s-1-2 n} \mathrm{~b}_{n}, \quad 0 \leqslant n \leqslant s-1, \\
E \mathrm{x}_{k}=-a[k][p-s-k] \mathrm{x}_{k-1}, \quad 0 \leqslant k \leqslant p-s-1 \quad\left(\mathrm{x}_{-1} \equiv 0\right), \\
E \mathrm{y}_{k}= \begin{cases}-a[k][p-s-k] \mathrm{y}_{k-1}, & 1 \leqslant k \leqslant p-s-1, \\
\mathrm{a}_{s-1}, & k=0,\end{cases} \\
E \mathrm{a}_{n}=a[n][s-n] \mathrm{a}_{n-1}, \quad 0 \leqslant n \leqslant s-1 \quad\left(\mathrm{a}_{-1} \equiv 0\right), \\
E \mathrm{~b}_{n}= \begin{cases}a[n][s-n] \mathrm{b}_{n-1}+\mathrm{a}_{n-1}, & 1 \leqslant n \leqslant s-1, \\
\mathrm{x}_{p-s-1}, & n=0,\end{cases}
\end{gathered}
$$




$$
\begin{aligned}
& F \mathrm{x}_{k}= \begin{cases}\mathrm{x}_{k+1}, & 0 \leqslant k \leqslant p-s-2, \\
\mathrm{a}_{0}, & k=p-s-1,\end{cases} \\
& F \mathrm{y}_{k}=\mathrm{y}_{k+1}, \quad 0 \leqslant k \leqslant p-s-1 \quad\left(\mathrm{y}_{p-s} \equiv 0\right), \\
& F \mathrm{a}_{n}=\mathrm{a}_{n+1}, \quad 0 \leqslant n \leqslant s-1 \quad\left(\mathrm{a}_{s} \equiv 0\right), \\
& F \mathrm{~b}_{n}= \begin{cases}\mathrm{b}_{n+1}, & 0 \leqslant n \leqslant s-2, \\
\mathrm{y}_{0}, & n=s-1 .\end{cases}
\end{aligned}
$$

\section{Колчан Кронекера}

\section{ПРИЛОЖКЕНИЕ Б}

Напомним основные понятия, относящиеся к колчанам [13], [20].

Б.1. Колчаны и их представления. Колчан $Q$ - это ориентированный граф, т.е. четверка $Q=(I, A, s, t)$, состоящая из конечного набора $I$ вершин, конечного набора $A$ ориентированных ребер (стрелок) и двух отображений $s$ и $t$ из $A$ в $I$. Ориентированное ребро $a \in A$ начинается в вершине $s(a)$ и заканчивается в $t(a)$.

Представление колчана $Q$ (над $\mathbb{C}$ ) - это набор конечномерных векторных пространств $V_{i}$ над $\mathbb{C}$, по одному для каждой вершины $i \in I$, и $\mathbb{C}$-линейных отображений $r_{i j}: V_{i} \rightarrow V_{j}$, по одному для каждого ориентированного ребра $\stackrel{i}{\bullet} \stackrel{j}{\rightarrow} \bullet \quad$ Размерность представления $\rho$ колчана $Q$ есть элемент из $\mathbb{Z}[I]$, определяемый размерностями $V_{i}$, $i \in I: \operatorname{dim}(\rho)=\sum_{i \in I} \operatorname{dim}_{\mathbb{C}}\left(V_{i}\right) i$.

Морфизм из представления $\rho$ колчана $Q$ в другое его представление $\rho^{\prime}$ есть $I$ градуированное $\mathbb{C}$-линейное отображение $\phi=\bigoplus_{i \in I} \phi_{i}: \bigoplus_{i \in I} V_{i} \rightarrow \bigoplus_{i \in I} V_{i}^{\prime}$, удовлетворяющее свойству $r_{i j}^{\prime} \phi_{i}=\phi_{j} r_{i j}$ для каждого ориентированного ребра $\stackrel{i}{\bullet} \stackrel{a}{\rightarrow} \bullet$. Это дает категорию представлений колчана $Q$, обозначаемую далее $\operatorname{Rep}(Q)$.

Если в колчане $Q$ нет ориентированных циклов, то классы изоморфизмов простых объектов в $\operatorname{Rep}(Q)$ находятся во взаимно однозначном соответствии с вершинами колчана $Q$. Простой объект, соответствующий вершине $i \in I$, задается векторными пространствами

$$
V_{j}= \begin{cases}\mathbb{C} & \text { при } j=i, \\ 0 & \text { в других случаях }\end{cases}
$$

и $\mathbb{C}$-линейными отображениями

$$
r_{i j}=0 \quad \text { для всех } i, j \in I \text {. }
$$

Говорят, что колчан имеет конечный тип, если лежащий в его основе неориентированный граф есть граф Дынкина конечного типа. Аналогично, колчан имеет аффинныц тип, если этот неориентированный граф есть граф Дынкина аффинного типа. Говорят, что колчан имеет однократные связи, если в нем нет пар вершин, соединенных более чем одной стрелкой. 
Б.2. Категория $\operatorname{Rep}(\mathbf{K})$ представлений колчана Кронекера. В колчане Кронекера $\mathbf{K}$ присутствуют две вершины, связанные двумя сонаправленными ребрами; другими словами, $\mathbf{K}=(\{0,1\},\{f, g\}, s, t)$, где $s(f)=s(g)=0$ и $t(f)=t(g)=1$ :

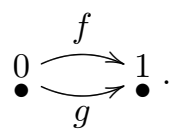

Представлением $\rho$ колчана $\mathbf{K}$ является четверка $\left(\left(V_{0}, V_{1}\right),\left(r_{01}, \bar{r}_{01}\right)\right)$, состоящая из двух $\mathbb{C}$-линейных пространств $V_{0}$ и $V_{1}$ и двух линейных отображений $r_{01}, \bar{r}_{01} \in$ $\operatorname{Hom}_{\mathbb{C}}\left(V_{0}, V_{1}\right)$. Размерность представления $\rho$ есть $\operatorname{dim}(\rho)=\left(\operatorname{dim}_{\mathbb{C}}\left(V_{0}\right), \operatorname{dim}_{\mathbb{C}}\left(V_{1}\right)\right)$. Простые объекты в категории $\operatorname{Rep}(\mathbf{K})$ задаются двумя представлениями $\rho_{0}=((\mathbb{C}, 0)$, $(0,0))$ и $\bar{\rho}_{0}=((0, \mathbb{C}),(0,0))$. Напомним классификацию неразложимых представлений колчана Кронекера $\mathbf{K}$, суммированную ниже в Б.2.1.

Имеется соответствие между неразложимыми представлениями колчана и множеством $\Delta_{+}$положительных корней алгебры Ли, соответствующей графу Дынкина, связанному с колчаном. Это соответствие является взаимно однозначным для колчанов конечного типа с однократными связями [26]. А именно, представление $\rho$ колчана $Q$ неразложимо, если и только если $\operatorname{dim}(\rho) \in \Delta_{+}$, и, обратно, для каждого $\alpha \in \Delta_{+}$, имеется с точностью до изоморфизма единственное неразложимое представление $\rho$ колчана $Q$ такое, что $\operatorname{dim}(\rho)=\alpha$.

Для колчанов аффинного типа биекция между классами изоморфизмов неразложимых представлений и положительными корнями соответствующей аффинной алгебры Ли имеет место только для положительных вещественных корней $\alpha \in \Delta_{+}^{\text {re }}$ [27]. Для всякого положительного мнимого корня $\alpha \in \Delta_{+}^{\mathrm{im}}$ существует несчетное множество $\operatorname{Ind}_{\alpha}$ неизоморфных неразложимых представлений, соответствующих $\alpha$. В действительности множество $\operatorname{Ind}_{\alpha}$ не зависит от $\alpha \in \Delta_{+}^{\mathrm{im}}[28]$. Если $\alpha \in \mathbb{Z}[I]$ не лежит в $\Delta_{+}$, то множество неразложимых представлений размерности $\alpha$ пусто.

Неориентированный граф, связанный с колчаном Кронекера $\mathbf{K}$, есть расширенный граф Дынкина $\mathrm{A}_{1}^{(1)}$. Как известно, $\alpha \in \Delta_{+}$является положительным вещественным корнем для $\mathrm{A}_{1}^{(1)}$, если $\alpha=(n+1, n)$ или $\alpha=(n, n+1)$, и $\alpha \in \Delta_{+}$является положительным мнимым корнем, если $\alpha=(n+1, n+1)$, где $n \in \mathbb{Z}_{+}$. В частности, $\alpha_{0}=(1,0)$ и $\bar{\alpha}_{0}=(0,1)$ - простые корни, а $\delta=(1,1)$ - первый мнимый корень. Простые корни $\alpha_{0}$ и $\bar{\alpha}_{0}$ отвечают соответствующим простым объектам $\rho_{0}=((\mathbb{C}, 0),(0,0))$ и $\bar{\rho}_{0}=((0, \mathbb{C}),(0,0))$ в категории $\operatorname{Rep}(\mathbf{K})$. Остальные вещественные корни находятся во взаимно однозначном соответствии с неразложимыми представлениями $\rho_{n}=\left(\left(\mathbb{C}^{n+1}, \mathbb{C}^{n}\right),(R, \bar{R})\right)$ и $\bar{\rho}_{n}=\left(\left(\mathbb{C}^{n}, \mathbb{C}^{n+1}\right),\left(R^{t}, \bar{R}^{t}\right)\right)$, где $R$ и $\bar{R}-$ прямоугольные матрицы,

$$
R=\left(\begin{array}{ccccc}
1 & 0 & \ldots & 0 & 0 \\
0 & 1 & \ldots & 0 & 0 \\
\ldots & \ldots & \ddots & \ldots & \ldots \\
0 & 0 & \ldots & 0 & 0 \\
0 & 0 & \ldots & 1 & 0
\end{array}\right), \quad \bar{R}=\left(\begin{array}{ccccc}
0 & 1 & \ldots & 0 & 0 \\
0 & 0 & \ldots & 0 & 0 \\
\ldots & \ldots & \ddots & \ldots & \ldots \\
0 & 0 & \ldots & 1 & 0 \\
0 & 0 & \ldots & 0 & 1
\end{array}\right)
$$


Первый мнимый корень $\delta$ соответствует семейству $\operatorname{Ind}_{\delta}$ неразложимых представлений $\left((\mathbb{C}, \mathbb{C}),\left(r_{01}, \bar{r}_{01}\right)\right)$ для некоторых $r_{01}, \bar{r}_{01} \in \operatorname{Hom}_{\mathbb{C}}(\mathbb{C}, \mathbb{C})$, параметризованных точками из $\mathbb{C P}^{1}$. Но для положительного мнимого корня $\alpha$ множество $\operatorname{Ind}_{\alpha}$ не зависит от $\alpha$, а потому $\operatorname{Ind}_{\alpha}=\mathbb{C P}^{1}$ для любого мнимого корня $\alpha$. Сводкой этих результатов является следующее хорошо известное предложение (см., например, [29]), впервые полученное в [30].

\section{Б.2.1. Предложение.}

1. Если $\alpha \notin \Delta_{+}$, то множество неразложимых представлений колчана $\mathbf{K}$ размерности а пусто.

2. Если $\alpha \in \Delta_{+}^{\mathrm{re}}$, то неразложимое представление колчана $\mathbf{K}$ размерности $\alpha$ есть либо представление $\rho_{n}$, если $\alpha=(n+1, n)$, либо представление $\bar{\rho}_{n}$, если $\alpha=(n, n+1), n \in \mathbb{Z}_{+}$.

3. Если $\alpha \in \Delta_{+}^{\mathrm{im}}$, то $\operatorname{Ind}_{\alpha}=\mathbb{C P}^{1}$ и неразложимые представления имеют размерность $(n+1, n+1)$ для некоторого $n \in \mathbb{Z}_{+}$.

Благодарности. Авторы благодарны С. Арики, Т. Миве и И. Тютину за полезные замечания, а также Ю. Фуксу за ценные обсуждения и литературные указания. Часть данной работы была написана во время пребывания авторов в университете Киото, и они благодарят Т. Миву за теплое гостеприимство. Данная работа была частично поддержана РФФИ (гранты № 04-01-00303, 05-02-17451) и Программой поддержки ведущих научных школ (грант № 1578.2003.2), а также РФФИ-ЯОПН (грант № 05-01-02934ЯФ_а).

\section{Список литературы}

[1] D. Kazhdan, G. Lusztig, J. Amer. Math. Soc., 6 (1993), 905; 949; 7 (1994), 335; 383.

[2] M. Finkelberg, Geom. and Funct. Anal., 6 (1996), 249; V. G. Turaev, Quantum Invariants of Knots and 3-Manifolds, Walter de Gruyter, Berlin-New York, 1994.

[3] G. Moore, N. Seiberg, "Lectures on RCFT", Superstrings'89, Proc. Spring School (Trieste, Italy, April 3-14, 1989), eds. M. Green, R. Iengo, S. Randjbar-Daemi, E. Sezgin, A. Strominger, World Scientific, Singapore, 1990, 263.

[4] J. Fuchs, I. Runkel, C. Schweigert, Nucl. Phys. B, 646 (2002), 353; hep-th/0204148.

[5] E. Frenkel, D. Ben-Zvi, Vertex Algebras and Algebraic Curves, Math. Surv. Monographs, 28, AMS, Providence, RI, 2001; B. Bakalov, A. A. Kirillov, Lectures on Tensor Categories and Modular Functors, AMS, Providence, RI, 2001; J. Fuchs, I. Runkel, C. Schweigert, Nucl. Phys. B, 678 (2004), 511; hep-th/0306164.

[6] J. Fuchs, I. Runkel, C. Schweigert, Ribbon categories and (unoriented) CFT: Frobenius algebras, automorphisms, reversions, math.CT/0511590.

[7] M. Miyamoto, A theory of tensor products for vertex operator algebra satisfying $\mathrm{C}_{2}$-cofiniteness, math.QA/0309350.

[8] Y.-Z. Huang, J. Lepowsky, L. Zhang, A logarithmic generalization of tensor product theory for modules for a vertex operator algebra, math.QA/0311235.

[9] J. Fuchs, On non-semisimple fusion rules and tensor categories, hep-th/0602051.

[10] H. G. Kausch, Phys. Lett. B, 259 (1991), 448.

[11] B. L. Feigin, A. M. Gainutdinov, A. M. Semikhatov, I. Yu. Tipunin, Commun. Math. Phys., 265 (2006), 47; hep-th/0504093.

[12] J. Fuchs, S. Hwang, A. M. Semikhatov, I. Yu. Tipunin, Commun. Math. Phys., 247 (2004), $713 ;$ hep-th/0306274. 
[13] M. Auslander, I. Reiten, S.O. Smalø, Representation Theory of Artin Algebras, Cambridge Studies Adv. Math., 36, Cambridge Univ. Press, Cambridge, 1995.

[14] M. A. I. Flohr, Int. J. Mod. Phys. A, 11 (1996), 4147; hep-th/9509166.

[15] T. Kerler, V.V. Lyubashenko, Non-Semisimple Topological Quantum Field Theories for 3-Manifolds with Corners, Lect. Notes in Math., 1765, Springer, Berlin, 2001.

[16] M. Flohr, M. R. Gaberdiel, J. Phys. A, 39 (2006), 1955; hep-th/0509075.

[17] V. Lyubashenko, Commun. Math. Phys., 172 (1995), 467; hep-th/9405167; "Modular properties of ribbon abelian categories", Proc. 2nd Gauss Symposium. Conference A: Mathematical and Theoretical Physics (Münich, Germany, August 2-7, 1993), eds. M. Behara, R. Fritish, R. G. Lintz, de Gruyter, Berlin, 1995, 529; hep-th/9405168; J. Pure Applied Algebra, 98 (1995), 279; V. Lyubashenko, S. Majid, J. Algebra, 166 (1994), 506.

[18] M. R. Gaberdiel, H. G. Kausch, Nucl. Phys. B, 477 (1996), 293; hep-th/9604026.

[19] S. MacLane, Homology, Springer, Berlin, 1963.

[20] W. Crawley-Boevey, Lectures on representations of quivers, Lectures in Oxford in 1992, available at http://www.amsta.leeds.ac.uk/ ${ }^{\sim}$ pmtwc/.

[21] H. G. Kausch, Nucl. Phys. B, 583 (2000), 513; hep-th/0003029.

[22] M. R. Gaberdiel, H. G. Kausch, Phys. Lett. B, 386 (1996), 131; hep-th/9606050.

[23] H. G. Kausch, Curiosities at $c=-2$, hep-th/9510149.

[24] S. Arkhipov, R. Bezrukavnikov, V. Ginzburg, J. Am. Math. Soc., 17 (2004), 595; math.RT/0304173.

[25] Y.-Z. Huang, L. Kong, Full field algebras, math.QA/0511328.

[26] P. Gabriel, Manuscripta Math., 6 (1972), 71; correction, ibid., 6 (1972), 309; И. Н. Бернштейн, И. М. Гельфанд, В. А. Пономарев, УМН, 28:2(170) (1973), 19.

[27] Л. А. Назарова, Изв. АН СССР, сер. матем., 37 (1973), 752; P. Donovan, M. R. Freislich, The representation theory of finite graphs and associated algebras, Carleton Math. Lect. Notes, No. 5, Ottawa, Ont., Canada, Carleton University, 1973.

[28] V. Dlab, C. M. Ringel, Mem. Am. Math. Soc., 173 (1976), 1.

[29] I. Frenkel, A. Malkin, M. Vybornov, Affine Lie algebras and tame quivers, math.RT/0005119.

[30] L. Kronecker, Sitzungsber. Akad. Berlin, 1890 (1890), 1225. 